\title{
Croissance cristalline en phase liquide : éléments théoriques.
}

\author{
Jean-Paul Garandet \\ CEA/DRT/DTEN/SMP/LESA, CEA-Grenoble \\ 17 rue des Martyrs, F-38054 GRENOBLE CEDEX, FRANCE
}

\section{INTRODUCTION}

\section{DE LA GERMINATION À LA CROISSANCE}

2.1. FORMATION DE LA PHASE SOLIDE

2.1.1. Stabilité d'un embryon solide

2.1.2. Distribution d'agrégats et germination

2.2. INTERFACES ET MÉCANISMES DE SOLIDIFICATION

2.2.1. Structure d'interface et équilibre thermodynamique local

2.2.2. Cinétiques de croissance

\section{DÉFAUTS CRISTALLINS}

3.1. DU DÉFAUT PONCTUEL AU GRAIN PARASITE

3.2. CONTRAINTES DE CROISSANCE

\section{SÉGRÉGATION SOLUTALE}

4.1. DIAGRAMMES DES PHASES

4.2. MÉCANISMES DE TRANSPORT

4.3. LOIS DE SÉGRÉGATION

4.3.1. Mélange complet dans le bain

4.3.2. Transport solutal diffusif

4.3.3. Coefficient de partage effectif

4.3.4. Striations de croissance

4.4. DÉSTABILISATION MORPHOLOGIQUE

\section{CONCLUSION : RETOUR VERS L'EXPÉRIENCE \\ REMERCIEMENTS \\ RÉFÉRENCES}

Mots-clés. Nucléation, cinétiques de croissance, dislocations, défauts cristallins, diagramme des phases, ségrégation solutale.

Élaboration et caractérisation des cristaux massifs et en couches minces pour l'optique

(C) EDP Sciences, 2003

http://dx.doi.org/10.1051/bib-sfo:2002809 


\section{INTRODUCTION}

La croissance cristalline à partir d'une phase liquide est longtemps restée affaire de praticiens, et les premiers efforts de formalisation scientifique dans ce domaine ne datent que des années 1950 . J.C. Brice pouvait fort justement écrire dans la préface de son livre paru en 1965 «Crystal growth is slowly changing from an art to a science » [1]. De nombreux progrès ont été réalisés depuis cette époque, par une utilisation fructueuse des formalismes de la thermodynamique et de la mécanique des milieux continus, et l'objet de ce texte est d'en faire un bref panorama. En effet, les concepts de la thermodynamique des systèmes à l'équilibre ont permis une meilleure compréhension des problèmes de stabilité et de cinétique de changement de phase, l'approche mécanique des milieux continus autorisant quant à elle un meilleur contrôle des champs de température et de concentration au cours du procédé de solidification. Le recours aux méthodes de simulation numérique, lié à l'augmentation de la puissance des moyens de calcul, a été un élément moteur des progrès enregistrés [2]. On peut sans trop de risques parier que cette évolution va se poursuivre, mais il faut bien garder à l'esprit que le rêve d'un processus de croissance cristalline entièrement automatisé relève encore de l'utopie.

Vu la complexité des problèmes et la diversité des disciplines scientifiques sous-jacentes, nous nous en tiendrons à une présentation élémentaire des concepts. Pour chaque domaine abordé, la bibliographie sera principalement restreinte aux papiers fondateurs ainsi qu'à des articles de synthèse et des ouvrages de référence récents. Il convient aussi d'insister sur le fait que les développements théoriques présentés sont basés sur des expériences de croissance cristalline de "petites » molécules (corps purs ou alliages binaires), souvent en liaison avec les problématiques de l'industrie des semiconducteurs (silicium, matériaux III-V et II-VI). Les besoins en matière de qualité cristalline pour ces matériaux étaient en effet devenus tels que les techniques empiriques habituelles ne permettaient plus de progresser efficacement, justifiant cet effort de compréhension théorique. Les résultats obtenus sont en principe applicables aux composés multi-constitués et aux macromolécules, sous réserve que des concepts de base (e.g. tensions d'interface, coefficients de partage...) puissent être définis sans ambiguiité.

Pour faire le lien avec le thème de cet ouvrage, il est nécessaire de rappeler que les matériaux pour l'optique sont souvent complexes et que l'adaptation des formalismes développés pour le cas des «petites » molécules n'est pas forcément évidente. Le problème se pose d'ailleurs de façon encore plus aiguë pour appliquer les résultats obtenus aux configurations de croissance des protéines et les autres molécules géantes de la biologie. En fait, on peut encore une fois envisager le problème sous un angle « économique » et considérer que si les techniques empiriques donnent satisfaction, il n'est pas besoin de se lancer dans des développements théoriques nécessairement lourds et finalement assez éloignés de la culture du praticien. L'avenir dira si la demande en terme de qualité cristalline en matériaux pour l'optique justifiera le développement de moyens théoriques importants.

Nous nous intéresserons d'abord (chapitre 2) au problème de la formation et de la croissance d'une entité solide se développant dans le liquide, en insistant particulièrement sur le concept de surfusion, qui apparaît comme le moteur de la transformation liquide-solide. Le chapitre $\underline{3}$ est consacré à une étude sur les défauts cristallins dont l'influence néfaste sur les propriétés d'usage des matériaux a été souvent mise en évidence. Le problème clé est ici celui des contraintes mécaniques à l'intérieur du cristal, et nous proposerons un certain nombre de solutions permettant l'amélioration de la qualité structurale des matériaux. Nous aborderons enfin au chapitre $\underline{4}$ les problèmes d'homogénéité chimique dans les alliages, pour montrer comment cette ségrégation est gouvernée par les phénomènes de transport de masse et de chaleur dans la phase liquide.

\section{DE LA GERMINATION À LA CROISSANCE}

\subsection{Formation de la phase solide}

La formation d'un agrégat solide susceptible de croître au sein d'un liquide est un problème fort complexe, et l'objet de notre présentation sera d'essayer de bien saisir le rôle des différents paramètres physiques. Pour plus d'informations sur le sujet, le lecteur intéressé pourra consulter l'article de synthèse de B. Mutaftschiev [ㅁ] ou l'ouvrage de W. Kurz et D. J. Fisher [4]]. Le phénomène clé est ici 
la distribution des fluctuations de densité au sein de la phase liquide, qui permettent de donner naissance à un noyau stable. Nous ne considérerons que le cas des corps purs, mais une théorie est également disponible pour l'étude des phénomènes de germination dans les alliages. Signalons aussi que nous discuterons des problèmes de stabilité des phases à partir du concept d'enthalpie libre, les variables de température et de pression étant a priori les plus importantes pour le suivi de la transformation liquide-solide. Il importe toutefois de rappeler que, pour les phases condensées auxquelles nous nous intéressons, énergie et enthalpie restent généralement très voisines sauf à considérer des pressions extrêmement élevées rarement utilisées en croissance cristalline. Concernant le vocabulaire, nous emploierons indifféremment les mots de germination et de nucléation pour désigner la formation d'un noyau cristallin, bien que le terme nucléation soit en fait un anglicisme adapté au français.

\subsubsection{Stabilité d'un embryon solide}

Le premier point à aborder est celui de la stabilité d'un agrégat solide, destiné à devenir le noyau d'un germe cristallin, en contact ou non avec une tierce phase pouvant catalyser la germination. On pourrait en effet penser que se placer au-dessous de la température de fusion du matériau suffit à garantir la stabilité, mais il ne faut pas oublier de considérer le terme énergétique associé à l'existence d'une tension d'interface liquide-solide $\sigma_{\mathrm{LS}}$. Nous allons d'abord considérer le cas où la phase solide et la phase liquide sont seules en présence, pour lequel on parle de germination homogène. La variation d'enthalpie libre du système liée à la formation d'un embryon solide sphérique de rayon $r$ peut se mettre sous la forme :

$$
\Delta G=4 / 3 \pi r^{3} \Delta G_{\mathrm{v}}+4 \pi r^{2} \sigma_{\mathrm{LS}} .
$$

La variation d'enthalpie libre par unité de volume $\Delta G_{\mathrm{v}}$ est négative pour $T$ inférieur à la température de fusion $T_{\mathrm{F}}$ du matériau, et correspond donc à un terme moteur pour le processus de solidification. En revanche, le terme associé à la formation d'une interface entre solide et liquide est toujours positif dans la mesure où $\sigma_{\mathrm{LS}}>0$ et il s'agit donc d'un terme frein pour la germination. On peut relier $\Delta G_{\mathrm{v}}$ à la différence $\left(T_{\mathrm{F}}-T\right)$ dénommée surfusion et à la chaleur latente de solidification $\Delta H_{\mathrm{v}}$. Pour ce faire, écrivons d'abord la relation d'équilibre thermodynamique à $T=T_{\mathrm{F}}$, i.e. $\Delta G_{\mathrm{v}}=\Delta H_{\mathrm{v}}-T_{\mathrm{F}} \Delta S_{\mathrm{v}}=0$. Pour une température $T<T_{\mathrm{F}}$, il vient :

$$
\Delta G_{\mathrm{v}}=\Delta H_{\mathrm{v}}-T \Delta S_{\mathrm{v}}=\left(T_{\mathrm{F}}-T\right) \Delta S_{\mathrm{v}}=\left(T_{\mathrm{F}}-T\right) \Delta H_{\mathrm{v}} / T_{\mathrm{F}} .
$$

La variation d'entropie volumique $\Delta S_{\mathrm{v}}$ est négative pour une transformation du liquide vers une phase solide plus ordonnée. L'expression ci-dessus a été obtenue en considérant $\Delta S_{\mathrm{v}}$ et $\Delta H_{\mathrm{v}}$ constantes dans l'intervalle $\left(T_{\mathrm{F}}-T\right)$, mais on peut dans une approche plus complète tenir compte des lois de variations des capacités calorifiques $C_{\mathrm{P}}^{\mathrm{S}}(T)$ et $C_{\mathrm{P}}^{\mathrm{L}}(T)$. La surfusion apparaît ici pour la première fois dans son rôle de moteur de la transformation liquide-solide.

Intéressons nous maintenant à la variation de $\Delta G$ (Éq. 1) en fonction du rayon de l'agrégat à une température $T<T_{\mathrm{F}}$ donnée. Le résultat, représenté sur la figure 1 , montre que pour les faibles dimensions le terme de surface en $r^{2}$ est dominant, ce qui se traduit par une augmentation de l'enthalpie libre du système. En revanche, aux dimensions plus élevées, le terme de volume en $r^{3}$ devient prépondérant, et l'enthalpie décroît. Il existe donc une barrière énergétique $\Delta G^{*}$, correspondant à un noyau critique de rayon $r^{*}=-2 \sigma_{\mathrm{LS}} / \Delta G_{\mathrm{v}}$ et à un nombre $n^{*}$ d'unités structurales, à franchir pour initier le processus de solidification. Dans les systèmes usuels où les calculs ont pu être effectués, le rayon critique comprend typiquement de cent à mille unités structurales (atomes ou molécules), mais l'application numérique doit toujours être faite avec les données thermodynamiques disponibles pour le composé à cristalliser. 


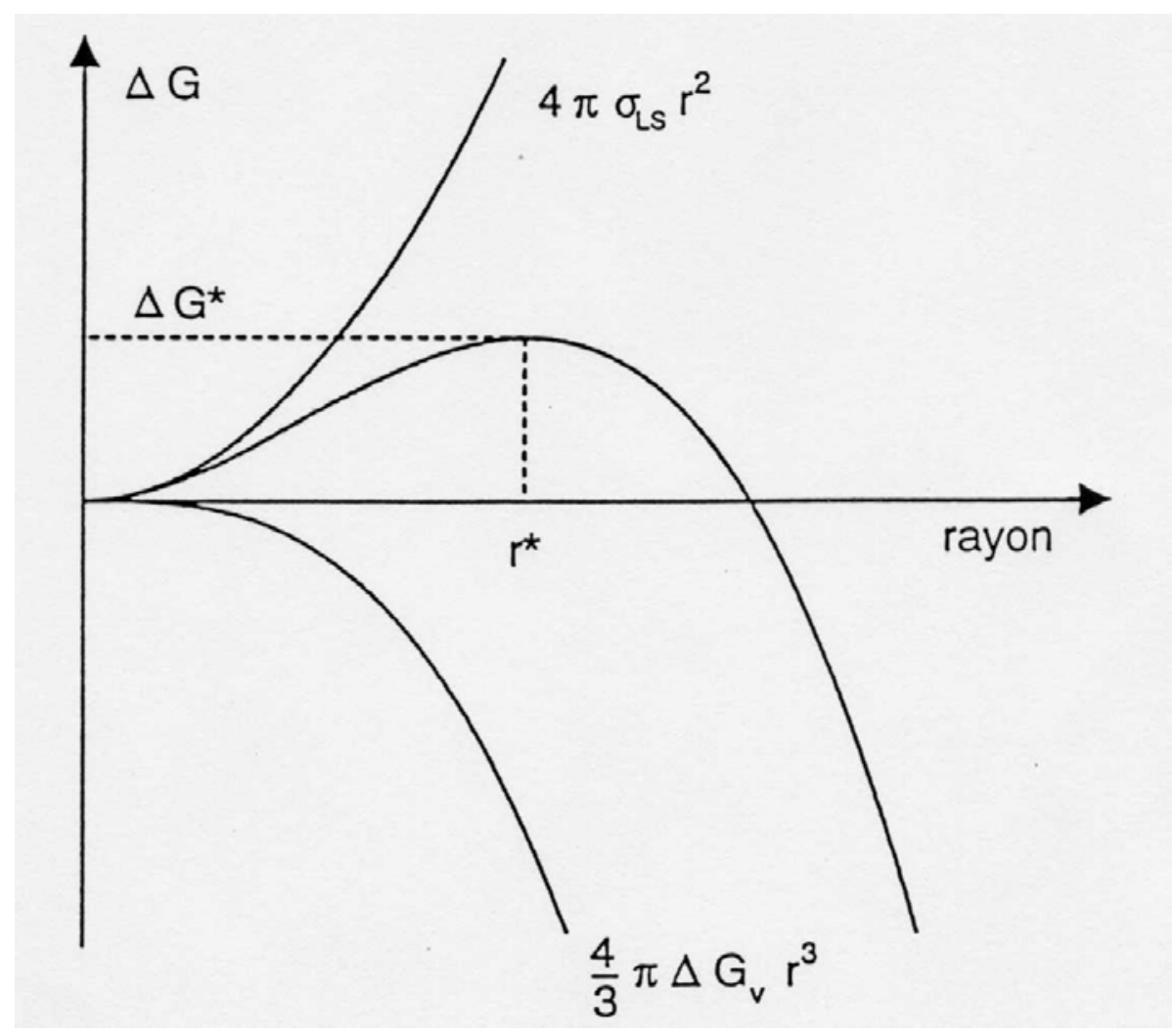

FIG. 1. Variation de l'enthalpie libre d'excès en fonction du rayon de l'embryon pour une configuration de germination homogène. Le maximum de la courbe, atteint au rayon critique $r^{*}$, représente la barrière $\Delta G^{*}$ à franchir pour initier la croissance de la phase solide.

Des résultats qualitativement similaires peuvent être obtenus dans le cas très important en pratique où un substrat (impureté ou paroi du creuset par exemple) va favoriser le processus de nucléation. On parlera alors de germination hétérogène, et il faut maintenant prendre en compte des diverses surfaces en présence (solide-liquide, solide-substrat et liquide-substrat), ainsi que des tensions d'interface associées. Il est intéressant de faire apparaître l'angle de raccord de la portion de sphère solide sur le substrat (appelé aussi angle de mouillage, voir Fig. 2) dans le calcul de l'énergie libre du système, ce qui permet d'exprimer les différentes données du problème sous la forme :

variation de l'enthalpie libre de volume de la portion de sphère solide : $4 / 3 \pi r^{3} f_{1}(\theta) \Delta G_{\mathrm{v}}$, énergie de formation d'une surface de contact solide-liquide : $4 \pi r^{2} f_{2}(\theta) \sigma_{\mathrm{LS}}$, énergie de formation d'une surface de contact solide-substrat : $\pi r^{2} \sin \theta \sigma_{\mathrm{S}-\mathrm{Sub}}$, énergie liée à la diminution de la surface de contact liquide-substrat : $-\pi r^{2} f_{2}(\theta) \sigma_{\mathrm{L}-\mathrm{Sub}}$, où les fonctions $f_{1}(\theta)$ et $f_{2}(\theta)$ sont respectivement définies par

$$
\begin{aligned}
& f_{1}(\theta)=1 / 4\left(2-3 \cos \theta+\cos ^{3} \theta\right), \\
& f_{2}(\theta)=1 / 2(1-\cos \theta) .
\end{aligned}
$$

Par rapport à la situation où le substrat est plongé dans le liquide, la variation d'enthalpie libre du système liée à la formation de l'embryon solide peut alors se mettre sous la forme :

$$
\Delta G=4 / 3 \pi r^{3} \Delta G_{\mathrm{v}} f_{1}(\theta)+4 \pi r^{2} \sigma_{\mathrm{LS}} f_{2}(\theta)+\pi r^{2} \sin ^{2} \theta\left(\sigma_{\mathrm{S}-\mathrm{Sub}}-\sigma_{\mathrm{L}-\mathrm{Sub}}\right) .
$$

En utilisant la relation de Young pour l'angle de contact $\left(\sigma_{\mathrm{S}-\mathrm{Sub}}+\sigma_{\mathrm{LS}} \cos \theta=\sigma_{\mathrm{L}-\mathrm{Sub}}\right)$ et l'identité mathématique $f_{2}(\theta)-1 / 4 \sin ^{2} \theta \cos \theta=f_{1}(\theta)$, l'expression de $\Delta G$ se transforme en :

$$
\Delta G=\left[4 / 3 \pi r^{3} \Delta G_{\mathrm{v}}+4 \pi r^{2} \sigma_{\mathrm{LS}}\right] f_{1}(\theta) \text {. }
$$




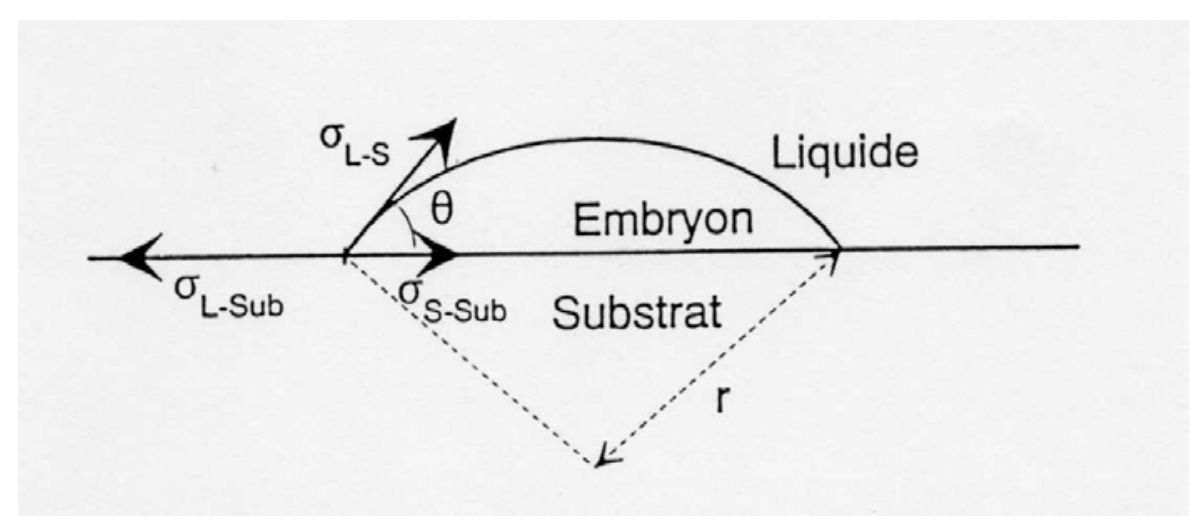

FIG. 2. Angle de mouillage $\theta$ et diverses tensions d'interface pour une configuration de germination hétérogène d'un embryon solide sur un substrat.

Le rayon critique, donné par la relation $\mathrm{d}\left(\Delta G_{\mathrm{het}}\right) / \mathrm{d} r=0$, est toujours $r^{*}=-2 \sigma_{\mathrm{LS}} / \Delta G_{\mathrm{v}}$, mais il est très important de comprendre que le nombre d'atomes du germe est maintenant réduit d'un facteur $f_{1}(\theta)$ par rapport au cas homogène, $n_{\text {het }} *=n^{*} f_{1}(\theta)$. Or ce facteur peut prendre des valeurs très inférieures à l'unité si le solide mouille bien le substrat, de 0,5 à $\theta=90^{\circ}$ à $1,7.10^{-4}$ pour $\theta=10^{\circ}$, en passant par $1,3.10^{-2}$ pour $\theta=30^{\circ}$. En revanche, pour le cas d'un solide non mouillant $\left(\theta=180^{\circ}\right)$, on retrouve $f_{1}(\theta)=1$ et la configuration de la germination homogène puisque le substrat ne joue aucun rôle dans l'apparition d'un embryon solide. L'équation (4) permet également de comprendre que la barrière énergétique est elle aussi réduite de ce facteur $f_{1}(\theta), \Delta G_{\mathrm{het}}{ }^{*}=\Delta G^{*} f_{1}(\theta)$. Nous serons amenés à revenir sur cette modification dans le cadre de la discussion sur l'efficacité des processus de nucléation dans la section suivante.

Les résultats précédents donnent la stabilité d'un agrégat sphérique pour une température donnée, mais n'indiquent pas à quelle température le processus de solidification va être initié. Il va nous falloir pour cela considérer la distribution statistique de ces agrégats dans le fluide, pour en déduire la densité d'embryons critiques, i.e. prêts à devenir des germes de solidification stables par incorporation d'une unité structurale.

\subsubsection{Distribution d'agrégats et germination}

Le problème est de calculer la distribution d'équilibre d'ensembles « solides » à $n$ atomes (les clusters) dans une unité de volume de liquide. Nous allons d'abord nous placer dans une configuration de germination homogène et considérer un modèle de solution idéale, i.e. sans enthalpie de mélange, de $N_{n}$ clusters à $n$ atomes avec $N_{1}$ atomes de liquide. L'étape suivante consiste à calculer l'enthalpie libre de la distribution, qui fait apparaître un terme entropique lié à la configuration des clusters dans la phase mère :

$$
\Delta G=N_{n} \Delta G_{n}-T \Delta S_{n}
$$

Le terme $\Delta G_{n}$ peut être exprimé en reformulant l'équation (1) pour faire apparaître le nombre $n$ d'atomes du cluster. Le terme d'entropie peut quant à lui se calculer à partir de la constante de Boltzmann $k$ et du nombre de possibilités de placer $N_{n}$ clusters dans $N_{1}$ atomes de liquide :

$$
\Delta S_{n}=k \ln \left[\left(N_{1}+N_{n}\right) ! /\left(N_{1} ! N_{n} !\right)\right] .
$$

On reporte ensuite le résultat précédent dans l'équation (므) en utilisant l'approximation de Stirling $(\ln N !=N \ln N-N)$, et on exprime la condition l'équilibre par $\mathrm{d}(\Delta G) / \mathrm{d} N_{n}=0$, ce qui conduit à :

$$
N_{n} /\left(N_{1}+N_{n}\right)=\exp \left(-\Delta G_{n} / k T\right) .
$$

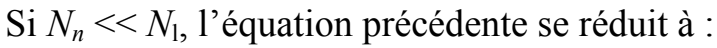

$$
N_{n} / N_{1}=\exp \left(-\Delta G_{n} / k T\right) .
$$


On parvient donc à un résultat relativement intuitif, à savoir que la distribution de clusters à $n$ atomes suit une loi d'Arrhenius fonction de leur enthalpie libre de formation. Il convient d'insister sur le fait que le résultat précédent ne peut être correct pour les valeurs négatives de $\Delta G_{n}$, pour lesquelles l'hypothèse $N_{n} \ll N_{1}$ n'est pas vérifiée. Cette limitation n'est cependant pas gênante pour notre propos, dans la mesure ou nous allons nous intéresser seulement aux clusters d'énergie critique $\Delta G^{*}$ (Fig. 1), i.e. à ceux dont l'enthalpie libre pourra baisser par adjonction d'une unité structurale supplémentaire. Plus précisément, nous allons faire l'hypothèse que le taux de germination peut être estimé à partir du produit du nombre $N^{*}$ de clusters critiques et de la probabilité $p$ d'incorporation d'une unité structurale sur un cluster critique.

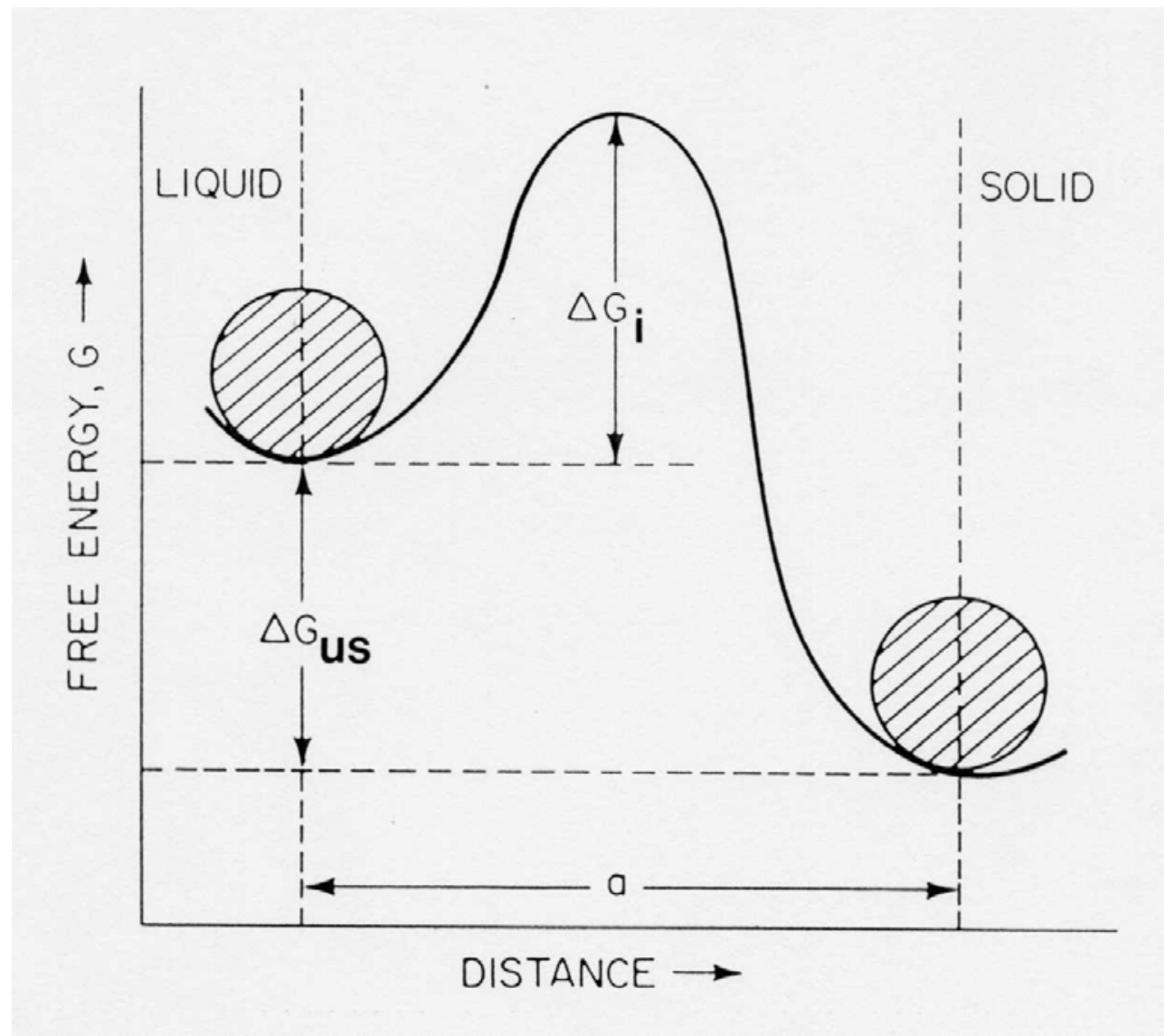

FIG. 3. Enthalpie libre d'une unité structurale à l'interface solide-liquide pour une température inférieure à la température de fusion [11]. L'incorporation dans le cristal se traduit par un gain d'amplitude $\Delta G_{\text {us }}$ mais nécessite le franchissement d'une barrière de hauteur $\Delta G_{i}$.

Nous allons de plus supposer que, en particulier pour des raisons d'encombrement stérique à l'interface solide-liquide, le processus d'adjonction nécessite le franchissement d'une barrière de potentiel (Fig. 3). On peut alors considérer que l'incorporation d'une unité structurale dans le réseau cristallin est un processus statistique dépendant de la hauteur $\Delta G_{i}$ de la barrière par une loi d'Arrhenius. Les autres variables pertinentes du problème sont le nombre $n_{\mathrm{S}}$ de sites disponibles en surface de l'embryon et la fréquence $f$ à laquelle l'unité structurale essaye de franchir la barrière. Nous prendrons en première approximation $n_{\mathrm{S}}=\eta\left(n^{*}\right)^{2 / 3}$, le facteur de forme $\eta$ étant de l'ordre de l'unité, et $f=k T / h$, ce qui conduit à l'expression du taux de germination :

$$
J=N_{1} \eta\left(n^{*}\right)^{2 / 3}(k T / h) \exp \left[-\left(\Delta G^{*}+\Delta G_{i}\right) / k T\right] .
$$

Il est d'usage d'isoler le terme en $\exp \left(-\Delta G^{*} / k T\right)$, et d'écrire l'équation (7) sous la forme :

$$
J=K_{\mathrm{v}} \exp \left(-\Delta G^{*} / k T\right) .
$$

Il est cependant préférable d'utiliser l'équation (ㄱ), car la forme de l'équation (ㅁ) tend à faire penser que le taux de germination augmente exponentiellement avec la surfusion, ce qui n'est pas exact du 
fait de la présence du terme en $\exp \left(-\Delta G_{i} / k T\right)$. De fait ce terme peut conduire à une limitation de caractère cinétique aux basses températures, le processus de formation des germes étant inhibé par la barrière de potentiel à franchir pour l'incorporation dans le solide. Cet effet peut même conduire à l'obtention de matériaux vitreux. Cela posé, et pour faire le lien avec la littérature sur le sujet, il est intéressant d'estimer l'ordre de grandeur de $K_{\mathrm{v}}$ en fonction des différents paramètres du problème :

$N_{1}$ ne dépend que des masses molaire $M$ et volumique $\rho$ du matériau, ainsi que du nombre d'Avogadro $\mathcal{N}, N_{1}=(\rho / M) \mathcal{N}$. Rappelons que, dans la mesure où nous avons travaillé sur une unité de volume, $N_{1}$ s'exprime en $\mathrm{m}^{-3}$. En prenant comme valeurs $M=200 \mathrm{~g} / \mathrm{mol}$, $\rho=2000 \mathrm{~kg} / \mathrm{m}^{3}$ et $\mathcal{N}=6.10^{23} \mathrm{~mol}^{-1}$, il vient $N_{1}=6.10^{27} \mathrm{~m}^{-3}$.

Le nombre d'atomes $n^{*}$ de l'embryon critique étant typiquement compris entre 100 et 1000, le nombre d'atomes de surface $n_{\mathrm{S}}=\eta\left(n^{*}\right)^{2 / 3}$ sera de l'ordre de quelques dizaines.

Le terme en $\exp (-\Delta G / k T)$ peut être estimé à partir d'expériences de mesure de coefficients de diffusion en phase liquide, et on trouve que dans la pratique $\exp \left(-\Delta G_{i} / k T\right)$ est de l'ordre de 0,01-0,05 ; en d'autres termes, l'unité structurale doit tenter plusieurs dizaines de sauts avant de réussir son incorporation dans le réseau cristallin.

La fréquence d'essai ne dépend des conditions expérimentales que par le biais de la température. En prenant $T=1000 \mathrm{~K}, k=1,4 \cdot 10^{-23}$ et $h=6,6 \cdot 10^{-34}$, il vient $k T / h=2.10^{13} \mathrm{~s}^{-1}$.

Dans le cadre de notre analyse des ordres de grandeur, il est licite de poser $\eta\left(n^{*}\right)^{2 / 3} \exp \left(-\Delta G_{i} / k T\right) \approx 1$, et on trouve tous calculs faits $K_{\mathrm{v}} \sim 10^{40} \mathrm{~m}^{-3} \cdot \mathrm{s}^{-1}$. Pour tenir compte des variations d'un système à l'autre, et en gardant à l'esprit les nombreuses hypothèses mises en jeu, nous écrirons $K_{\mathrm{v}} \sim 10^{40+/-3} \mathrm{~m}^{-3} \cdot \mathrm{s}^{-1}$.

D'un point de vue expérimental, le déclenchement du processus de nucléation se traduit par un dégagement de chaleur latente de changement de phase qui peut être mesuré, ce qui permet de définir la surfusion de germination. Pour fixer un ordre de grandeur, les surfusions nécessaires pour atteindre la nucléation homogène sont souvent de l'ordre de $20 \%$ de la température de fusion exprimée en Kelvin, mais il faut pour cela limiter les contacts entre l'échantillon et le creuset. Dans la pratique, nous allons voir que le mécanisme de germination hétérogène est souvent favorisé. Pour ce faire, il suffit de reprendre les équations (ㅁ)-( $\underline{8})$ en remplaçant $N_{1}$ par $N_{\text {het }}$, densité volumique de particules nucléantes et $\Delta G^{*}$ par $\Delta G_{\text {het }}{ }^{*}$, barrière énergétique de formation de la portion de sphère sur le substrat. Il faut également substituer $\left(n_{\text {het }}{ }^{*}\right)^{2 / 3}$ à $\left(n^{*}\right)^{2 / 3}$, mais nous supposerons que cette modification importe peu au vu des ordres de grandeur mis en jeu. Le taux de germination hétérogène peut donc s'écrire :

$$
J_{\text {het }}=N_{\text {het }} \eta\left(n_{\text {het }}{ }^{*}\right)^{2 / 3}(k T / h) \exp \left[-\left(\Delta G_{\text {het }}{ }^{*}+\Delta G_{i}\right) / k T\right]=K_{\text {het }} \exp \left(-\Delta G_{\text {het }} * / k T\right) \text {. }
$$

Les taux de germination hétérogène et homogène se présentent donc sous des formes qualitativement similaires, mais les ordres de grandeur sous jacents sont très différents : pour estimer $K_{\text {het }}$, en prenant $N_{\text {het }}=10^{18} \mathrm{~m}^{-3}$ et en gardant $\eta\left(n_{\text {het }}{ }^{*}\right)^{2 / 3} \exp \left(-\Delta G_{i} / k T\right)=1$ et $k T / h=2.10^{13} \mathrm{~s}^{-1}$, il vient $K_{\text {het }} \sim 10^{31} \mathrm{~m}^{-3} \cdot \mathrm{s}^{-1}$. En pratique, $K_{\text {het }}$ est toujours inférieur à $10^{35} \mathrm{~m}^{-3} \cdot \mathrm{s}^{-1}$, et peut prendre des valeurs beaucoup plus faibles si le système offre peu de centres nucléants. Cela dit, si le terme pré-exponentiel est très fortement réduit, la barrière énergétique est elle aussi significativement abaissée ( $c f$. le facteur $f_{1}(\theta)$ de l'équation (4)), et la comparaison de l'efficacité des mécanismes homogène et hétérogène n'est pas évidente a priori.

Dans la pratique, c'est la mesure de la surfusion de germination par le dégagement de chaleur latente lié à la recalescence qui permet de trancher. La procédure est de supposer un processus de nucléation homogène et d'en déduire un préfacteur $K_{\mathrm{v}}$ expérimental. Si le $K_{\mathrm{v}}$ ainsi mesuré est inférieur à $10^{35} \mathrm{~m}^{-3} \cdot \mathrm{s}^{-1}$, l'hypothèse de départ conduit à une contradiction, et on peut affirmer que le processus s'est déroulé de façon hétérogène. De façon générale, on peut se risquer à affirmer que l'effet de réduction de la barrière énergétique sera dominant dès que le liquide sera en contact avec un substrat bien mouillé par le solide. De fait, la germination hétérogène peut être un problème délicat à contrôler pour les expériences de croissance cristalline, dans la mesure où des grains parasites peuvent se former, mais on peut également tirer bénéfice des propriétés de mouillage : c'est par exemple le cas dans l'industrie de l'aluminium, où l'on introduit volontairement des particules germinantes pour favoriser la formation de grains de petite taille. 
Nous venons de présenter brièvement la théorie dite «classique» de la germination, séduisante par sa relative simplicité, mais dont les hypothèses sont parfois difficiles à justifier. Par exemple, l'utilisation du formalisme de la thermodynamique est souvent discutable, en particulier pour le cas de la germination hétérogène où le nombre d'atomes du germe critique peut être très faible. Depuis le travail original de Turnbull et Fisher [5] de nombreux développements ont été proposés pour fonder la théorie sur une base plus physique (voir par exemple [ㅁ-ㅁ]), mais la difficulté est que ces modèles font souvent apparaître des constantes physiques difficilement accessibles, surtout pour le cas de systèmes complexes tels que ceux des matériaux pour l'optique. On peut donc affirmer que la théorie classique fournit un cadre robuste à l'interprétation des mécanismes de germination [9], au moins pour la base de données expérimentale dont nous disposons à ce jour. D'un point de vue pratique, retenons la nécessité de soigner la thermique du dispositif de solidification, notamment pour éviter la présence de points froids susceptibles de donner naissance à des grains parasites.

\subsection{Interfaces et mécanismes de solidification}

Nous allons maintenant quitter le domaine des surfusions élevées et des dimensions nanométriques pour nous intéresser au processus d'attachement des unités structurales sur une phase cristalline «macroscopique » pour lequel des surfusions de l'ordre du kelvin sont généralement suffisantes. Il nous faut cependant d'abord préciser un certain nombre d'idées sur les notions de structure d'interface et d'équilibre thermodynamique local. Ici encore, nous nous en tiendrons aux phénomènes de base, une présentation beaucoup plus complète et rigoureuse pouvant être trouvée dans l'article de revue de P. Bennema [10] ou dans le livre de M. C. Flemmings [11].

\subsubsection{Structure d'interface et équilibre thermodynamique local}

La surface de séparation entre la phase liquide et la phase solide (l'interface) peut être plus ou moins complexe : la géométrie la plus simple à concevoir est celle d'un plan parfaitement lisse à l'échelle atomique, on parle alors d'interface facettée (Fig. 4-a) mais on peut également imaginer une configuration de plus ou moins grande rugosité (Fig. 4-b). On peut enfin concevoir que, dans la pratique, une interface facettée au niveau macroscopique présente souvent des défauts de structure à l'échelle de l'unité structurale (terrasses, marches, décrochements, trous ou unités adsorbées, $c f$. Fig. 4-c), et nous verrons que ces rugosités locales jouent en fait un grand rôle dans la cinétique du processus de solidification. Signalons également que des modèles théoriques ont été proposés pour prédire la rugosité des interfaces (voir par exemple le travail de base de K. Jackson [12]), mais que leur présentation dépasserait le cadre de ce texte.

Cela dit, la notion même de surface de séparation est une commodité mathématique dont la validité physique mérite d'être discutée. Les résultats de simulation par dynamique moléculaire montrent en effet que l'amplitude des mouvements des unités structurales ne subit pas nécessairement une discontinuité marquée au passage de la température de fusion. La transition liquide-solide se fait parfois sur une zone diffuse dont l'épaisseur est typiquement de quelques unités structurales (Fig. 5). Les problèmes de configuration et de cinétique de croissance sur ces interfaces diffuses ont été abordés dans la littérature (voir par exemple le travail fondateur de J.W. Cahn [13]), mais les développements théoriques dépassent le cadre de notre propos. Nous nous en tiendrons donc à la représentation simplifiée de surface matérielle pour caractériser les fronts solide-liquide.

Le processus de croissance cristalline se déroulant très fréquemment en présence de gradients de température et de concentration, le système ne peut pas être globalement à l'équilibre thermodynamique. On peut néanmoins introduire la notion d'équilibre thermodynamique local à l'interface de croissance, en étudiant sous quelles conditions une unité structurale incorporée dans le solide pourra trouver une «bonne» position dans le réseau cristallin. Quelle que soit la structure du front de croissance, on peut penser que le processus nécessitera une diffusion de "surface» de coefficient $D_{\mathrm{S}}$ sur une échelle de longueur de l'ordre de la dimension de l'unité structurale, notée ici $a$. Le problème a été volontairement formulé de façon assez vague, mais nous allons voir que l'analyse des ordres de grandeur suffit pour tirer une conclusion sur la validité de l'hypothèse d'équilibre local. 


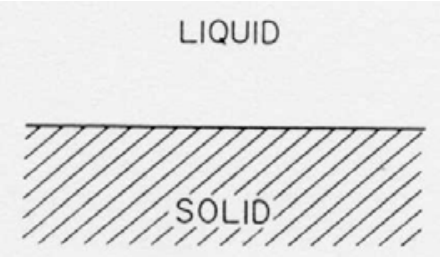

a

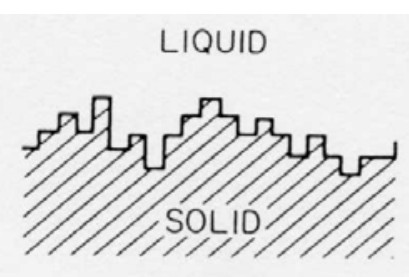

b

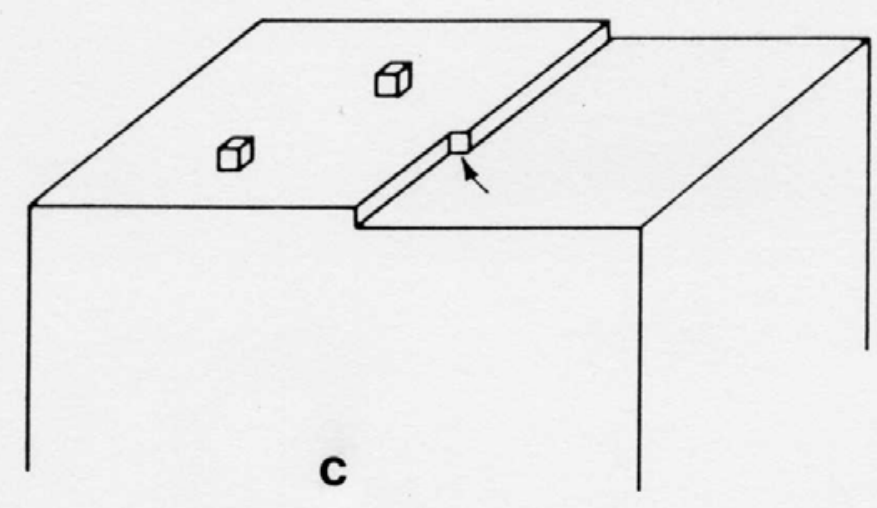

FIG. 4. Surfaces de séparation facettées (a,c) et rugueuse (b) entre phases solide et liquide [11]. Par rapport au cas idéal (a), l'interface (c) présente deux unités adsorbées, une marche et un décrochement.

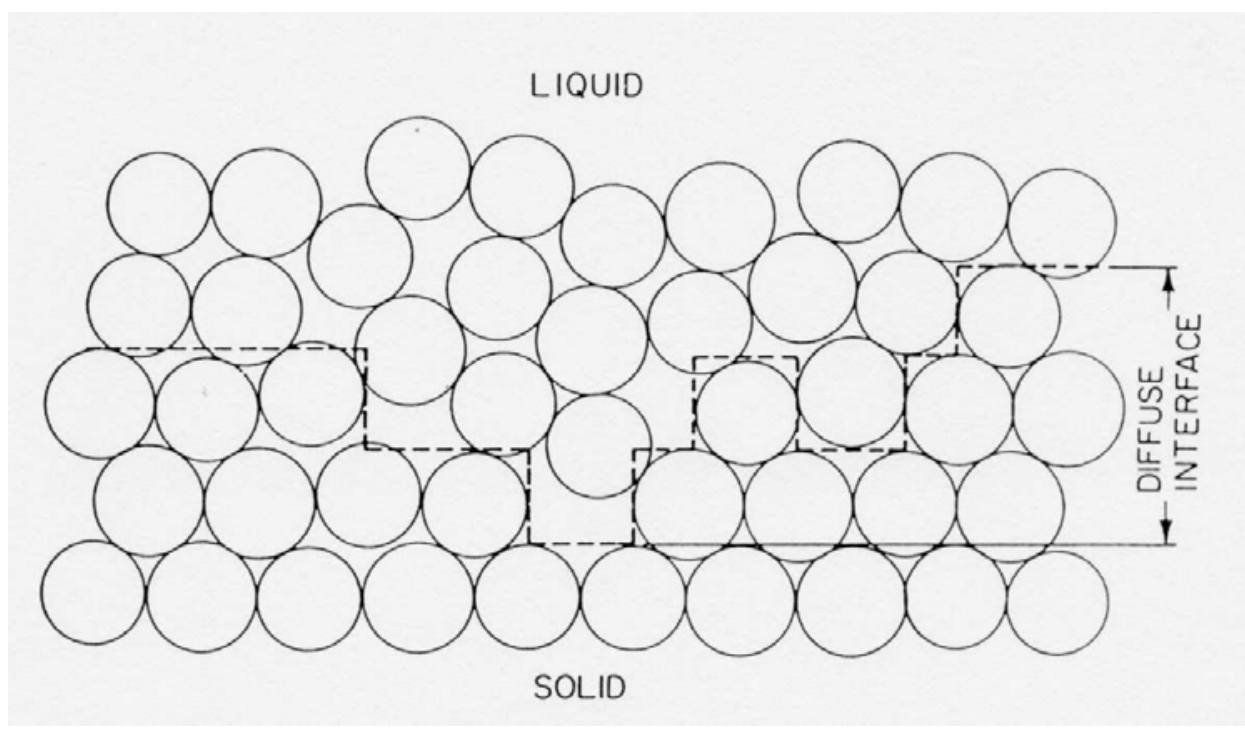

FIG. 5. Représentation de la transition liquide-solide à travers une zone diffuse, dont l'épaisseur est typiquement de quelques unités structurales.

Un résultat général des études sur les phénomènes de transport est qu'un processus de diffusion de coefficient $D$ sur une échelle $\delta$ se fait à une vitesse de l'ordre de $D / \delta$. Pour savoir si notre unité structurale aura le temps de se positionner dans le réseau cristallin, nous pouvons donc comparer la vitesse de diffusion $D_{\mathrm{S}} / a$ et la vitesse d'avancée du front de croissance $V_{\mathrm{I}}$, en calculant un nombre de Peclet, $P e=V_{\mathrm{I}} / V_{\mathrm{D}}=a V_{\mathrm{I}} / D_{\mathrm{S}}$. À partir de valeurs typiques, $D_{\mathrm{S}}=5.10^{-10} \mathrm{~m}^{2} \cdot \mathrm{s}^{-1}, a=5.10^{-10} \mathrm{~m}$ et $\mathrm{V}_{\mathrm{I}}=10^{-5} \mathrm{~m} \cdot \mathrm{s}^{-1}$, on obtient $P e=10^{-5}$. On constate donc que pour prendre en défaut l'hypothèse d'équilibre local à l'interface, il faut travailler à des vitesses de solidification très élevées de l'ordre du $\mathrm{m} . \mathrm{s}^{-1}$. De telles valeurs ne sont jamais rencontrées dans les expériences de croissance cristalline, mais le sont dans certains processus métallurgiques, par exemple dans l'élaboration de verres métalliques. 


\subsubsection{Cinétiques de croissance}

Il importe de comprendre que l'incorporation d'une unité structurale sur l'interface n'est pas nécessairement thermodynamiquement favorisée, la question étant ici encore de savoir si l'accroissement de l'énergie interfaciale peut être compensé par un gain d'enthalpie de volume. Le paramètre clé est ici la configuration géométrique du système, et plus précisément le nombre de voisins de l'unité structurale incidente. Au vu de notre présentation des phénomènes de germination, on peut concevoir que l'incorporation sera plus difficile (i.e. nécessitera une surfusion plus élevée) sur une interface plane que sur une interface rugueuse. Nous allons maintenant examiner très brièvement les différents mécanismes permettant la croissance sur les divers types d'interface.

Le cas le plus simple d'un point de vue mathématique est celui de l'interface rigoureusement plane, puisque l'on peut adapter à deux dimensions le formalisme développé pour l'étude des phénomènes de germination homogène. Reprenons brièvement les grandes lignes de la démarche, le premier point étant l'expression de l'enthalpie libre de formation d'un noyau cylindrique monocouche sur l'interface :

$$
\Delta G=\pi r^{2} a \Delta G_{\mathrm{v}}+2 \pi r a \sigma_{\mathrm{LS}},
$$

$a$ représentant encore une dimension caractéristique de l'unité structurale. La condition d'extremum définissant le rayon $r^{*}$ du germe critique est toujours donnée par $\mathrm{d}(\Delta G) / \mathrm{d} r=0$, ce qui conduit à $r^{*}=-\sigma_{\mathrm{LS}} / \Delta G_{\mathrm{v}}$ et permet de définir une barrière énergétique $\Delta G_{2 \mathrm{D}} *$. Le résultat du calcul de la densité d'équilibre de clusters critiques $N_{2 \mathrm{D}}{ }^{*}$ à $n_{2 \mathrm{D}}{ }^{*}$ unités structurales peut se mettre sous la forme $N_{2 \mathrm{D}}{ }^{*} / N_{\mathrm{S}}=\exp \left(-\Delta G_{2 \mathrm{D}} * / k T\right), N_{\mathrm{S}}=1 / a^{2}$ représentant le nombre de sites d'attache potentiels par unité de surface, et le taux de germination bidimensionnel s'exprime par :

$$
J_{2 \mathrm{D}}=N_{\mathrm{S}} \eta\left(\mathrm{n}_{2 \mathrm{D}} *\right)^{1 / 2}(k T / h) \exp \left[-\left(\Delta G_{2 \mathrm{D}} *+\Delta G_{i}\right) / k T\right] .
$$

Remarquons que le coefficient de facteur de forme $\eta$ reliant le nombre d'unités structurales en périphérie et $n_{2 \mathrm{D}}{ }^{*} \mathrm{n}$ 'a aucune raison d'être le même que pour le processus tridimensionnel, mais on peut en revanche penser que la barrière énergétique $\Delta G_{i}$ reste quant à elle identique. La dernière étape de la démonstration consiste à exprimer la vitesse de croissance par le produit du nombre de noyaux formés par unité de temps par le volume de l'unité structurale :

$$
V_{2 \mathrm{D}}=J_{2 \mathrm{D}} a^{3} \text {. }
$$

L'expression ci-dessus est relativement satisfaisante d'un point de vue intellectuel, mais conduit à la difficulté que la croissance ne peut avoir lieu qu'à des surfusions importantes. En effet, au voisinage du point de fusion, le nombre d'unités structurales dans un hypothétique cluster critique est très élevé, typiquement de l'ordre de $10^{4}-10^{5}$, et la construction d'un germe par des fluctuations de densité en phase liquide est très improbable. Le problème est que les résultats expérimentaux montrent qu'une surfusion de quelques degrés est largement suffisante pour permettre la solidification. Nous sommes donc amenés à penser que, dans la pratique, la croissance sur les interfaces facettées se fait par un mécanisme alternatif plus efficace que la germination bidimensionnelle.

Nous allons bientôt revenir sur ce point, mais il nous faut d'abord présenter brièvement le processus de croissance sur une interface rugueuse, où la solidification peut être vue comme un processus purement cinétique. Considérons en effet le problème thermodynamique résolu, au sens où l'adjonction d'une unité structurale supplémentaire ne modifie pas la surface de contact solide-liquide. C'est donc le produit de la fréquence d'agitation thermique par la probabilité d'incorporation, déjà introduit au paragraphe 2.1.2, qui va gouverner la vitesse $V$ d'avancée de l'interface. Le nombre de transitions efficaces par unité de temps peut donc se mettre sous la forme :

$$
v_{\mathrm{L}-\mathrm{S}}=(k T / h) \exp \left[-\Delta G_{i} / k T\right] \text {. }
$$

Dans le domaine des faibles surfusions qui nous intéresse, la probabilité de transition du solide vers le liquide, bien que défavorisée thermodynamiquement, n'est pas négligeable, et on peut écrire de façon similaire :

$$
v_{\mathrm{S}-\mathrm{L}}=(k T / h) \exp \left[-\left(\Delta G_{\mathrm{us}}+\Delta G_{i}\right) / k T\right]
$$


$\Delta \mathrm{G}_{\text {us }}$ représentant la variation d'enthalpie libre liquide-solide pour une unité structurale, que l'on peut estimer par :

$$
\Delta G_{\mathrm{us}}=a^{3} \Delta G_{\mathrm{v}},
$$

la variation d'enthalpie libre liquide-solide par unité de volume ayant été introduite dans les équations (1)-(2). Chaque transition efficace se traduisant par une avancée ou un recul du front sur une distance caractéristique de la taille de l'unité structurale a, la vitesse de croissance sur une interface rugueuse $V_{\mathrm{R}}$ peut donc s'exprimer par :

$$
V_{\mathrm{R}}=a\left(v_{\mathrm{L}-\mathrm{S}}-v_{\mathrm{S}-\mathrm{L}}\right)=a(k T / h) \exp \left(-\Delta G_{\mathrm{i}} / k T\right)\left[1-\exp \left(-\Delta G_{\mathrm{us}} / k T\right)\right] .
$$

Dans le cas des faibles surfusions où $\Delta G_{\text {us }} / k T$ reste très petit devant l'unité, on peut utiliser l'approximation $1-\exp \left(-\Delta G_{\mathrm{us}} / k T\right) \approx\left(\Delta G_{\mathrm{us}} / k T\right)$. De par la relation de proportionnalité entre $\Delta G_{\mathrm{v}}$ et la surfusion $\Delta T$ proposée en équation (2), $V_{\mathrm{R}}$ peut se mettre sous la forme :

$$
V_{\mathrm{R}}=K_{\mathrm{R}} \Delta T \text {. }
$$

En prenant $a=5.10^{-10} \mathrm{~m}, k T / h=2.10^{13} \mathrm{~s}^{-1}(T=1000 \mathrm{~K}), \exp \left(-\Delta G_{i} / k T\right)=0,02$ et $\Delta H_{\mathrm{v}}=10^{9} \mathrm{~J} / \mathrm{m}^{3}$, et en utilisant la relation $\Delta G_{\text {us }}=a^{3} \Delta G_{\mathrm{v}}$, on trouve que $K_{\mathrm{R}}$, exprimé en $\mathrm{ms}^{-1} \cdot \mathrm{K}^{-1}$, est typiquement d'ordre de grandeur unité. Cela signifie que les vitesses de croissance raisonnables pour la cristallogenèse des matériaux à interface rugueuse correspondront toujours à des surfusions très faibles, résultat cohérent avec l'hypothèse conduisant à l'équation (14).

Revenons maintenant au problème des interfaces facettées dont nous avons vu qu'elles présentent souvent des défauts de structure, tels que marches et décrochements. L'idée de base est que ce sont ces défauts qui gouvernent la cinétique de solidification, dans la mesure où ils jouent le rôle de « rugosités locales » et donc de sites d'incorporation préférentiels. De fait, la croissance se fait latéralement, i.e. le long d'une direction parallèle à l'interface (Fig. 6), avec une vitesse $V_{\mathrm{L}}$ de type rugueux, $V_{\mathrm{L}} \sim \Delta T$. L'étape suivante est de supposer que la cinétique de croissance $V_{\mathrm{F}}$ normale d'une interface facettée dépend linéairement du produit de $V_{\mathrm{L}}$ par la densité de sites d'incorporation, dont le calcul dépasse malheureusement assez largement le cadre de ce texte.

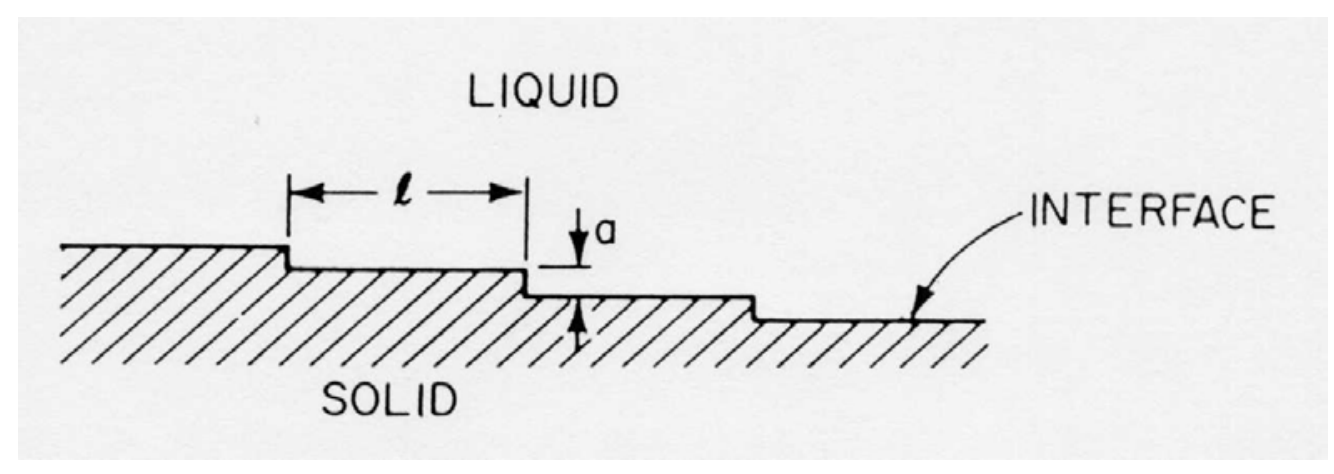

FIG. 6. Croissance latérale le long d'une direction parallèle à l'interface [11]. La vitesse de solidification est directement liée à la distance $l$ entre sites d'incorporation préférentiels et à la taille $a$ de l'unité structurale.

Le problème de la génération de défauts à caractère rugueux est en effet un problème très complexe, mais le lecteur intéressé pourra consulter le papier de référence de Burton, Cabrera et Frank [14] sur le sujet. Nous mentionnerons seulement que les lignes d'émergence de dislocations de type vis (voir section 3-1) sont une source de sites d'incorporation privilégiés, et que la densité de dislocations est donc un paramètre clé de la cinétique de solidification. On peut même montrer que la distance latérale entre deux lignes de sites d'incorporation voisines varie en $\Delta T^{-1}$ ou, de façon équivalente, que la densité de sites est proportionnelle à $\Delta T$. La dépendance en surfusion de la cinétique de croissance sur une interface facettée est donc de la forme :

$$
V_{\mathrm{F}}=K_{\mathrm{F}} \Delta T^{2} \text {, }
$$

la constante de proportionnalité $K_{\mathrm{F}}$ dépendant entre autres de la densité de dislocations. Signalons enfin qu'une interface facettée à l'équilibre ou aux faibles vitesses de solidification devient rugueuse 
aux fortes surfusions, dans la mesure où les processus cinétiques sont très ralentis. Une unité structurale rejoignant le front de croissance ne pourra que très difficilement repartir en phase liquide, même si sa position d'impact est thermodynamiquement défavorisée. On parle alors de «kinetic roughening » pour traduire cet état de fait.

Pour conclure sur ce bref panorama des cinétiques de croissance, il importe tout d'abord de préciser que les prédictions des équations (14) et (15) ont été vérifiées sur un grand nombre de matériaux modèles. S'agissant d'un domaine d'étude assez fondamental, il est compréhensible que les chercheurs se soient rarement risqués à travailler sur des matériaux complexes ou difficiles à mettre en oeuvre, comme ceux rencontrés dans les matériaux pour l'optique. Un autre élément est que, pour les configurations de croissance où la vitesse est imposée par l'expérimentateur (méthodes Bridgman ou Czochralski par exemple), ces études ont un caractère assez académique. En revanche, pour les techniques de croissance en solution, les problèmes de cinétique et de contrôle de la surfusion sont d'une importance capitale dans la pratique.

\section{DÉFAUTS CRISTALLINS}

L'ordre à grande distance au sein du cristal étant à la base des propriétés d'usage du matériau, on conçoit que toute rupture de cet ordonnancement puisse être source de problèmes. La présence de défauts peut avoir des conséquences variées telles que l'échauffement localisé sous rayonnement pour les matériaux laser ou la création de niveaux intermédiaires dans la bande interdite pour les semiconducteurs utilisés en optoélectronique. On peut par exemple montrer que les dislocations sont des centres de recombinaison non radiatifs qui dégradent fortement l'efficacité optique du matériau. $\mathrm{Ce}$ point est illustré en figure $\underline{7}$ sur un ensemble de diodes GaAlAs:Si élaborées par épitaxie en phase liquide [15]. Nous ne traiterons dans ce paragraphe que des défauts de structure, les problèmes liés à l'homogénéité chimique dans les matériaux multi-constitués faisant l'objet de la section $\underline{4}$.

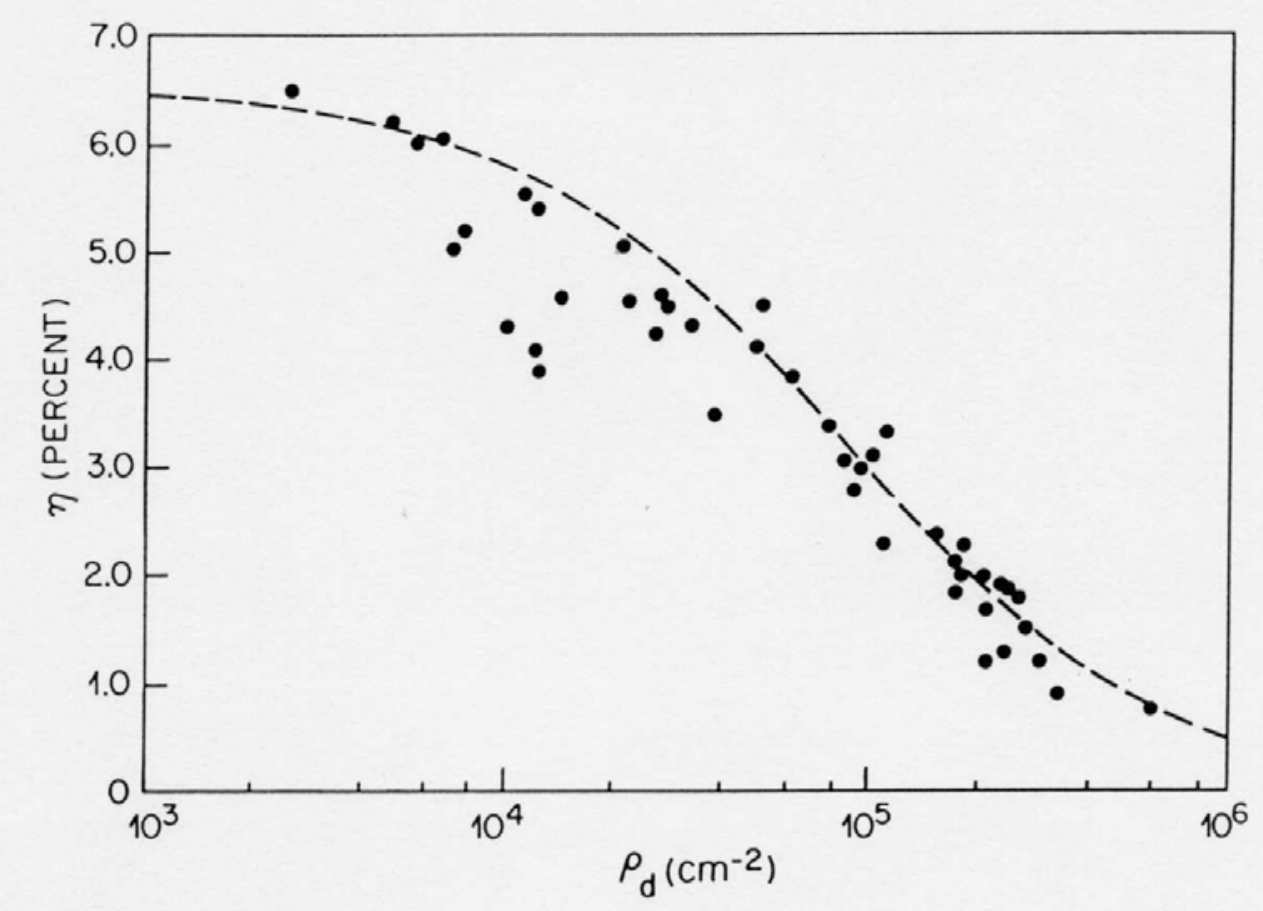

FIG. 7. Variation de l'efficacité radiative de 45 diodes GaAlAs:Si en fonction de la densité de dislocations dans la couche épitaxiée [15].

Nous allons d'abord proposer une classification dimensionnelle des différents défauts rencontrés dans les matériaux cristallins, en nous attachant particulièrement à discuter pour chaque type de défaut des causes liées à la mise en oeuvre du procédé de croissance. Il existe en effet des défauts de structure à caractère intrinsèque, au sens où leur formation est gouvernée par la 
thermodynamique des systèmes à l'équilibre, et qui seront donc inévitables en pratique. Cela dit, nous verrons aussi que les conditions expérimentales peuvent être optimisées pour permettre une amélioration de la qualité cristalline, notamment en limitant les contraintes mécaniques pendant la croissance et le refroidissement.

\subsection{Du défaut ponctuel au grain parasite}

Les défauts ponctuels peuvent être organisés en terme de complexité : les plus simples sont les lacunes (sites vacants du réseau) et les interstitiels (unités structurales présentes à des positions autres que celles nominalement prévues dans le réseau). Dans les composés de formule $\mathrm{AB}$, on trouve également les antisites (unité $\mathrm{A}$ en position $\mathrm{B}$ ou réciproquement) et les complexes (unité $\mathrm{A}$ en position $\mathrm{B}$ associée à une lacune de A) par exemple. Ce schéma peut être généralisé aux systèmes multiconstitués, le nombre de combinaisons possibles augmentant rapidement avec le nombre d'éléments présents dans le matériau. L'existence de ces défauts crée du désordre dans la structure cristalline, et permet d'abaisser l'enthalpie libre de la configuration par effet entropique : ces défauts existent donc à l'équilibre thermodynamique, dans une proportion en première approximation calculable à partir de leur énergie d'excès par un facteur d'Arrhenius.

Les impuretés, liées par exemple à la propreté de l'installation ou à la purification des matériaux de départ, sont pour leur part des défauts ponctuels considérés comme extrinsèques. Cette dénomination est en fait abusive, car il est facile de montrer que l'introduction d'une unité structurale étrangère dans un réseau conduit à un effet entropique significatif : si $N$ représente le nombre de sites d'accueil potentiels, l'entropie sera réduite d'un facteur $k T \ln (N)$, et cette contribution permettra souvent d'abaisser l'enthalpie libre de la configuration. On comprend tout de même que la présence d'impuretés en quantités "importantes " (typiquement à des fractions molaires supérieures à $10^{-6}$ ) dépende de la qualité du travail de l'expérimentateur. Cela dit, le problème de la purification des matériaux de départ est parfois loin d'être évident dans la pratique.

Les dislocations sont des défauts linéaires, dont les types principaux sont la vis (Fig. 8-a) et le coin (Fig. 8-b). Elles peuvent être la conséquence d'une coalescence de lacunes, mais leur formation est principalement liée à la présence de contraintes mécaniques associées aux gradients de température et de concentration dans le matériau. En effet, un cristal à l'équilibre thermodynamique ne devrait pas contenir de dislocations, et c'est l'énergie d'excès des contraintes qui permet la création des défauts. Limiter ces contraintes est donc un objectif capital pour l'expérimentateur, surtout pour les matériaux comme les semi-conducteurs III-V ou II-VI où la présence de dislocations se révèle particulièrement pénalisante pour le fonctionnement des dispositifs comme nous l'avons vu en figure $\underline{7}$. Nous reviendrons bientôt (section $\underline{3-2}$ ) en détail sur ce point.

Les macles sont des défauts de surface caractérisés par une rupture de l'ordonnancement des plans atomiques. Soit par exemple une orientation où le réseau cristallin du matériau est constitué d'une succession de trois types de plans $\alpha, \beta$ et $\gamma$ dans l'ordre nominal... $\alpha \beta \gamma \alpha \beta \gamma \alpha \beta \gamma \ldots$ Une macle sera caractérisée par un plan de symétrie miroir $\chi$ correspondant à l'ordonnancement ... $\alpha \beta \gamma \alpha \beta \not \beta \alpha \gamma \beta \alpha \ldots$ Comme dans le cas des dislocations, la configuration du cristal maclé n'est pas thermodynamiquement favorisée. Plusieurs mécanismes ont été invoqués pour expliquer l'apparition des défauts, mais une conclusion générale n'est pas évidente à tirer. Signalons seulement que pour un matériau de structure cristalline donnée, un paramètre physique important est l'énergie de faute d'empilement, dont la valeur dépend de l'orientation par le biais du nombre de liaisons perturbées.

Les défauts de volume sont principalement de deux types, grains parasites et inclusions, qui tous deux peuvent avoir un caractère soit thermodynamique soit lié à la mise en oeuvre du procédé de croissance. Par exemple, pour certains composés à diagramme des phases rétrograde (voir section 4-1), la formation d'inclusions liquides en cours de refroidissement par refusion du matériau élaboré est inévitable. Cela dit, les cristaux solidifiés peuvent également contenir des inclusions provenant de l'atmosphère du dispositif de croissance ou de particules étrangères présentes dans la phase liquide, cas pour lesquels l'expérimentateur possède des moyens d'action en contrôlant l'atmosphère du dispositif ou les conditions de solidification. On sait en effet que la vitesse de croissance est un paramètre clé pour l'incorporation ou le rejet des particules étrangères [16]. 


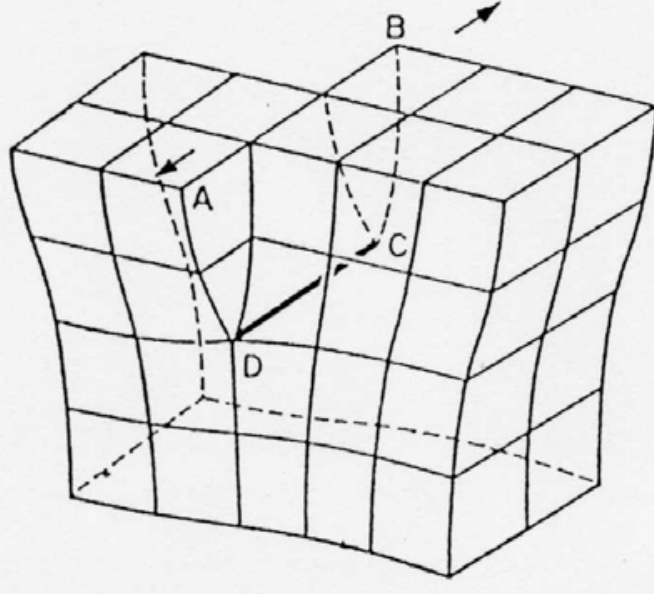

a

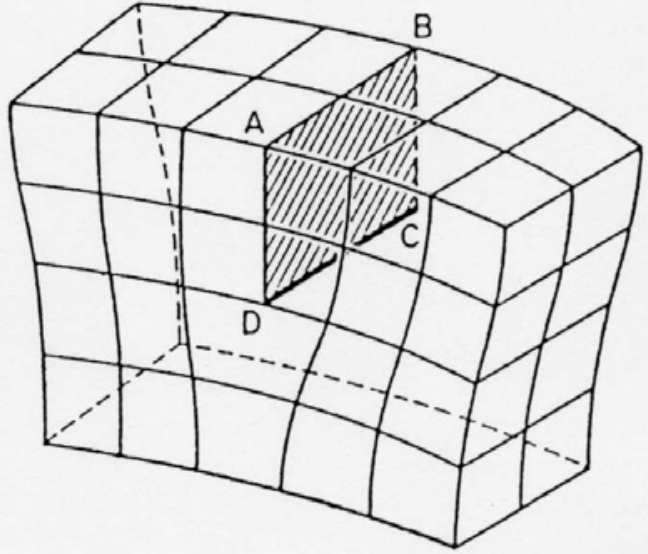

b

FIG. 8. Représentation schématique de dislocations vis (a) et coin (b). Par rapport au cas du cristal parfait, la surface $(A B C D)$ de défaut peut être soit un demi-plan de cisaillement (a), soit un demi-plan en excès (b).

Le cas des grains parasites est plus ambigu : leur formation par germination hétérogène (voir section 2-1) sur les parois du creuset ou sur des particules étrangères présentes dans le bain a souvent un caractère thermodynamique, mais il appartient à l'expérimentateur de contrôler les éventuels points froids au voisinage du front de solidification et la propreté de son installation. Des grains parasites peuvent également apparaître sur l'interface de croissance elle-même, par exemple au voisinage d'une bulle ou d'un précipité incorporé. Dans ce cas, l'énergie nécessaire à la création du défaut peut être d'origine élastique, mais des phénomènes de germination hétérogène peuvent également être envisagés. Signalons enfin qu'un réseau de dislocations peut s'organiser pour former des joints de grains faiblement désorientés, ce qui représente encore une forme de défaut tridimensionnel.

Ce bref panorama des défauts cristallins, et en particulier de ceux à caractère extrinsèque, nous a permis de mettre en évidence l'effet des contraintes mécaniques sur la qualité structurale du matériau élaboré. Ce point a fait l'objet de nombreuses études dans la littérature, et le lecteur est invité à consulter l'article de revue de Völk1 [17] pour plus d'informations sur le sujet. Nous allons maintenant examiner plus en détail le problème des contraintes liées à la présence de gradients de température et de concentration dans le matériau, mais il ne faut pas oublier que l'existence de zones de contact entre le cristal et un creuset, en solidification Bridgman par exemple, est également source de contraintes. Il peut de fait être intéressant de mettre en œuvre des stratégies d'encapsulation [18] permettant de limiter ces zones de contact, qui sont des endroits privilégiés pour la germination de dislocations, macles et grains parasites. Pour en terminer avec les défauts extrinsèques, rappelons également que les problèmes de propreté ne sont pas forcément évidents à gérer dans la pratique.

\subsection{Contraintes de croissance}

Un cristal en cours de croissance et de refroidissement est généralement soumis à des gradients de température provenant de la nécessité de canaliser le flux de chaleur. Vu la complexité du problème, nous ne ferons que mentionner au chapitre $\underline{4}$ quelques éléments sur le calcul de la thermique des dispositifs de croissance, mais il importe de bien garder à l'esprit à ce stade que le processus de solidification se déroule généralement en présence de gradients de température. De plus, les corps purs étant rarement les plus intéressants du point de vue des propriétés d'application, les matériaux d'usage sont généralement des composés multi-constitués et les phénomènes de ségrégation chimique que nous allons étudier au chapitre $\underline{4}$ font que les cristaux sont souvent le siège de gradients de concentration. Notre objectif dans ce paragraphe sera de montrer comment les contraintes associées à ces gradients vont activer les mécanismes de formation des dislocations. Nous nous restreindrons en effet au cas des 
dislocations, défauts qui, comme nous l'avons déjà signalé, se trouvent être particulièrement pénalisants dans les matériaux semiconducteurs III-V ou II-VI pour l'optoélectronique.

La clé du problème est la variation du paramètre de maille avec la température et la concentration. Nous allons montrer qu'un cristal soumis à ce type de gradients sera généralement contraint. Considérons pour ce faire un matériau isotrope dans une configuration à symétrie cylindrique $(r, \theta, z)$, et écrivons les composantes radiale, orthoradiale et axiale de la loi de Hooke isotrope reliant contrainte et déformation :

$$
\begin{aligned}
& \sigma_{r}=[E /(1+v)(1-2 v)]\left[(1-v) e_{r}+v e_{\theta}+v e_{z}-(1+v)\left(\alpha_{\mathrm{T}}\left(T-T_{0}\right)+\alpha_{\mathrm{C}}\left(C-C_{0}\right)\right)\right], \\
& \sigma_{\theta}=[E /(1+v)(1-2 v)]\left[v e_{r}+(1-v) e_{\theta}+v e_{z}-(1+v)\left(\alpha_{\mathrm{T}}\left(T-T_{0}\right)+\alpha_{\mathrm{C}}\left(C-C_{0}\right)\right)\right], \\
& \sigma_{z}=[E /(1+v)(1-2 v)]\left[v e_{r}+v e_{\theta}+(1-v) e_{z}-(1+v)\left(\alpha_{\mathrm{T}}\left(T-T_{0}\right)+\alpha_{\mathrm{C}}\left(C-C_{0}\right)\right)\right],
\end{aligned}
$$

où $E, v, \alpha_{\mathrm{T}}, \alpha_{\mathrm{C}}, T_{0}$ et $C_{0}$ représentent respectivement le module de Young, le coefficient de Poisson, les coefficients de dilatation linéaire thermique et solutal du matériau et une température et une concentration de référence, les déformations dans les directions radiale, orthoradiale et axiale étant notées $e_{r}, e_{\theta}$ et $e_{z}$. La question fondamentale est de prévoir si les déformations élastiques peuvent accommoder l'effet des termes $\alpha_{\mathrm{T}}\left(T-T_{0}\right)$ et $\alpha_{\mathrm{C}}\left(C-C_{0}\right)$. Pour un cristal à symétrie cylindrique, on peut montrer [19] que seules les variations linéaires de température et de concentration autorisent une solution à $\sigma_{\mathrm{r}}=\sigma_{\theta}=\sigma_{\mathrm{z}}=0$ des équations d'équilibre. Dans tous les autres cas, le cristal sera nécessairement contraint.

Intéressons nous maintenant à l'effet des contraintes sur un cristal initialement parfait. En deçà du seuil de limite élastique, ces contraintes seront accommodées de façon réversible, les déformations pouvant être calculées par la loi de Hooke modifiée, voir l'équation (16). Au-delà du seuil en revanche, une dégradation irréversible du matériau va se produire, avec la formation de dislocations pour relaxer les contraintes. Le comportement d'un cristal contenant initialement des défauts est moins facile à appréhender. On peut cependant se risquer à dire qu'en deçà d'un seuil empirique, la Critical Resolved Shear Stress (CRSS) dans la terminologie anglo-saxonne [20], la contrainte est accommodée de façon élastique et donc réversible. Au-delà de la CRSS en revanche, dans le régime dit "plastique ", la contrainte aura pour conséquence la mise en mouvement des dislocations et des effets irréversibles.

Ce mouvement nécessite le franchissement d'une barrière de potentiel d'énergie d'activation $U$ et la vitesse $V$ d'une dislocation peut également être reliée au niveau de contrainte par une relation empirique de la forme :

$$
V=\alpha_{1} \exp (-U / k T) \sigma_{\mathrm{e}}{ }^{n}
$$

Dans l'expression précédente, les constantes $\alpha_{1}$ et $n$ doivent être ajustées au moyen d'expériences dédiées, et $\sigma_{\mathrm{e}}$ représente la contrainte efficace, i.e. celle ayant un effet moteur pour le mouvement. L'étape suivante consiste à écrire une équation de bilan sur la densité de dislocations $N(\mathbf{r}, t)$, en écrivant que sa variation temporelle est proportionnelle à la vitesse moyenne des défauts $V$ :

$$
\mathrm{d} N(\mathbf{r}, t) / \mathrm{d} t=\alpha_{2} N(\mathbf{r}, t) \sigma_{\mathrm{e}} V,
$$

où $\alpha_{2}$ est encore une constante empirique. L'expression précédente revient à postuler que l'augmentation du nombre de dislocations est proportionnelle à leur densité, au chemin qu'elles parcourent et à la contrainte efficace. Cette hypothèse peut se justifier par des mécanismes physiques tels que le moulin de Frank-Read dont le développement dépasse notre propos (voir [21,22] pour une discussion plus complète). En tout état de cause, la relation choisie dépend fortement du type de matériau considéré. Une fois le modèle complété par une relation constitutive reliant contrainte efficace et contrainte appliquée, la densité de dislocations peut donc être en principe calculée (voir par exemple [23]) par intégration des équations (17) et (18).

Ce modèle « visco-plastique » peut être complété pour tenir compte de phénomènes tels que l'annihilation des dislocations au voisinage du front de solidification ou des interactions entre les différents systèmes de glissements, mais les raffinements conceptuels sont assez coûteux en terme de complexité. En ce sens, la notion de contrainte critique Critical Resolved Shear Stress permet souvent une première approche du niveau de contrainte tolérable pour la formation des défauts. Insistons sur le 
fait que la CRSS n'est pas une propriété intrinsèque du matériau, puisqu'elle dépend entre autres de la densité initiale de dislocations, et qu'à ce titre le modèle visco-plastique a certainement un caractère plus physique même s'il contient un certain nombre de paramètres empiriques. Dans la pratique, il appartient à l'expérimentateur de juger du niveau de compréhension nécessaire à la production d'échantillons de qualité structurale satisfaisante pour les applications d'usage.

Signalons pour terminer que les niveaux de contraintes dans les cristaux issus du procédé d'élaboration sont souvent élevés, la création de dislocations pendant la phase de croissance se traduisant par l'existence de contraintes dans le matériau à température ambiante. Une conséquence pratique en est que la manipulation et le conditionnement des cristaux sont souvent problématiques. Pour pallier cette difficulté, on utilise parfois des procédures de recuit à des températures élevées (typiquement entre 0,5 et $0,8 T_{\mathrm{F}}$ ). La mise en mouvement des dislocations peut en effet permettre l'annihilation de paires à vecteur de Burgers opposé ou sur la surface du cristal et ainsi réduire le niveau de contraintes. Cela posé, l'activation du mécanisme de glissement peut aussi conduire à une dégradation de la qualité structurale du matériau ! L'efficacité de la procédure de recuit est donc souvent jugée a posteriori, même si le modèle visco-plastique peut en principe fournir des prédictions intéressantes sur les problèmes de recuit.

D'un point de vue pratique, limiter les contraintes en cours d'élaboration et de refroidissement est souvent un objectif clé pour l'expérimentateur. Il est relativement facile de s'approcher de la configuration idéale de gradient de température uniforme dans les techniques de Bridgman ou de Gradient Freeze. En revanche, les cristaux Czochralski sont généralement plus contraints, dans la mesure où le contrôle des flux de chaleur dans le dispositif est beaucoup plus délicat. Un autre moyen d'améliorer la qualité structurale des cristaux élaborés est d'utiliser des éléments d'alliage jouant le rôle d'obstacles qui ralentissent le mouvement des dislocations, limitant par la même leur multiplication.

Concernant les gradients de concentration, l'étude des problèmes d'homogénéité solutale va faire l'objet du chapitre $\underline{4}$, mais on peut déjà signaler qu'il sera virtuellement impossible d'éviter les contraintes dans les systèmes concentrés. Le problème est souvent particulièrement aigu à la reprise sur germe, où des variations de composition importantes se font sur des distances très courtes. Pour conclure sur ces problèmes de qualité cristalline, il importe de rappeler que c'est à l'utilisateur du matériau de spécifier la densité de défauts tolérables dans le matériau élaboré, et que les exigences peuvent être très variables selon les applications visées.

\section{SÉGRÉGATION SOLUTALE}

La composition chimique des matériaux intéressants d'un point de vue des applications est souvent complexe, et il nous faut maintenant nous intéresser aux problèmes de l'homogénéité des cristaux élaborés. La maîtrise du procédé de croissance passe d'abord par la délimitation des domaines de stabilité des différentes phases solide et liquide en présence dans un espace température-concentration. Cela posé, la connaissance du diagramme des phases du système considéré est insuffisante pour contrôler les phénomènes de ségrégation chimique, et nous verrons que la compréhension des mécanismes de transport de masse est également nécessaire. Pour ce faire, nous reviendrons sur la formulation du problème avant de proposer quelques lois simples permettant d'estimer les variations de concentration dans les cristaux élaborés. Nous terminerons ce bref panorama des phénomènes de ségrégation en abordant les phénomènes de déstabilisation morphologique du front de croissance, dont les conséquences en termes de limitation de la vitesse de solidification sont très importantes.

\subsection{Diagrammes des phases}

Nous supposerons d'abord que les arguments présentés en section 2-2 pour discuter de la validité de l'hypothèse d'équilibre thermodynamique local au front de croissance peuvent être adaptés au cas des composés multi-constitués. Une conséquence importante pour notre propos est la possibilité de définir les plages de stabilité des différentes phases par minimisation de l'énergie libre du système. Si une telle procédure est appliquée au cas d'un composé semi-conducteur binaire comme le $\mathrm{CdTe}$, on obtient un diagramme des phases similaire à celui représenté en figure $\underline{9}$ [24], qui fait apparaître une zone de miscibilité complète à l'état liquide à haute température, un segment de droite représentant le composé défini stœchiométrique et deux courbes définissant les lieux de coexistence des phases liquide et 
solide. Considérons maintenant un alliage composé à $60 \%$ de $\mathrm{Cd}$ et $40 \%$ de Te (pourcentages atomiques) porté à une température de $1100{ }^{\circ} \mathrm{C}$ où le système est à l'état liquide, et imaginons un processus de refroidissement du système. Le diagramme des phases nous apprend que le matériau reste à l'état liquide jusqu'à une température légèrement supérieure à $1030^{\circ} \mathrm{C}$, lieu d'apparition de la première phase solide si les phénomènes de surfusion sont négligeables.

Il est capital de remarquer que le solide formé est de composition stœchiométrique CdTe, donc plus riche en tellure que le liquide lui ayant donné naissance. La solidification va donc se traduire par un enrichissement en cadmium de la solution. Si la température du système continue de baisser, ce processus d'enrichissement en cadmium va se poursuivre, la composition de la solution évoluant le long de la courbe de liquidus séparant le domaine liquide du domaine biphasé. Sur l'exemple présenté en figure $\underline{9}$, le domaine solide est réduit à un segment de droite, et le matériau élaboré va rester stœchiométrique, du moins jusqu'à l'apparition d'un composé eutectique formé d'une phase CdTe et d'une phase $\mathrm{Cd}$ à une température de $321^{\circ} \mathrm{C}$. Le processus de solidification se déroulerait de façon qualitativement similaire pour une solution initiale riche en tellure, avec formation de $\mathrm{CdTe}$ stœchiométrique et enrichissement de la phase liquide en tellure jusqu'à formation d'un composé eutectique $(\mathrm{CdTe}, \mathrm{Te})$ à une température de $446{ }^{\circ} \mathrm{C}$.

Dans la pratique, la situation est généralement plus complexe, même pour un composé binaire. Considérons en effet l'agrandissement de la région au voisinage du point de fusion du CdTe présenté en figure 10 [25], et imaginons le refroidissement d'un liquide de composition stœchiométrique. Ce nouveau diagramme des phases nous indique que le premier solide formé sera très légèrement plus concentré en Te que la solution, et que la composition du solide formé au cours du refroidissement va maintenant évoluer le long de la courbe du solidus. Les modifications induites par rapport au problème discuté à partir du diagramme des phases de la figure $\underline{9}$ sont a priori insignifiantes, mais leurs conséquences peuvent être dramatiques. En effet, pour un matériau semi-conducteur comme le CdTe, les propriétés électriques sont très sensibles à des variations minimes de composition. Par ailleurs, la courbure du solidus conduit à la formation de gouttes de tellure dans la matrice de CdTe à partir d'une température de l'ordre de $1300 \mathrm{~K}$, puisque le Te incorporé va se retrouver en quantité trop importante dans le matériau.

Une autre modification importante par rapport au cas précédent est que le domaine de stabilité de la phase solide présente une certaine étendue, on parle dans ce cas de zone de solubilité. La conséquence pratique en est que des matériaux élaborés à partir de solutions respectivement riches en tellure et en cadmium n'auront plus la même composition avec encore une fois des conséquences importantes en matière de propriétés électriques. Sur l'exemple présenté en figure $\underline{10}$, la zone de solubilité est très réduite, mais on peut comprendre qu'un composé défini ne soit pas strictement stœchiométrique, la contribution entropique permettant de stabiliser une configuration de composition légèrement déséquilibrée. Cette propriété de solubilité en phase solide est de fait très commune dans la nature, et il existe même des alliages où elle est observée sur l'ensemble le diagramme des phases. C'est par exemple le cas du système quasi-binaire GaSb-InSb (Fig. 11) et on parle alors de miscibilité complète [르].

Dans la pratique, la probabilité de trouver un diagramme des phases fiable dans la littérature décroît très rapidement avec le nombre de constituants de l'alliage. Il existe cependant un certain nombre de données disponibles pour les systèmes ternaires riches en composés potentiellement intéressants au niveau des applications, citons par exemple dans le cas des matériaux pour l'optique la section $\mathrm{Li}_{2} \mathrm{O}-\mathrm{B}_{2} \mathrm{O}_{3}$ du diagramme $(\mathrm{Li}, \mathrm{B}, \mathrm{O})$ [27] représentée en figure $\underline{12}$. Il importe de bien comprendre que cette figure permet d'évaluer directement la faisabilité de mise en oeuvre des techniques de croissance classiques pour l'élaboration d'un composé donné : on voit ainsi que les méthodes Bridgman et Czochralski sont a priori utilisables pour le composé à fusion congruente $\mathrm{Li}_{2} \mathrm{~B}_{4} \mathrm{O}_{7}$, mais que la cristallisation du composé $\mathrm{LiB}_{3} \mathrm{O}_{5}$ ne pourra se faire qu'à partir d'une solution riche en $\mathrm{B}_{2} \mathrm{O}_{3}$. 


\section{Cd-Te Phase Diagram}

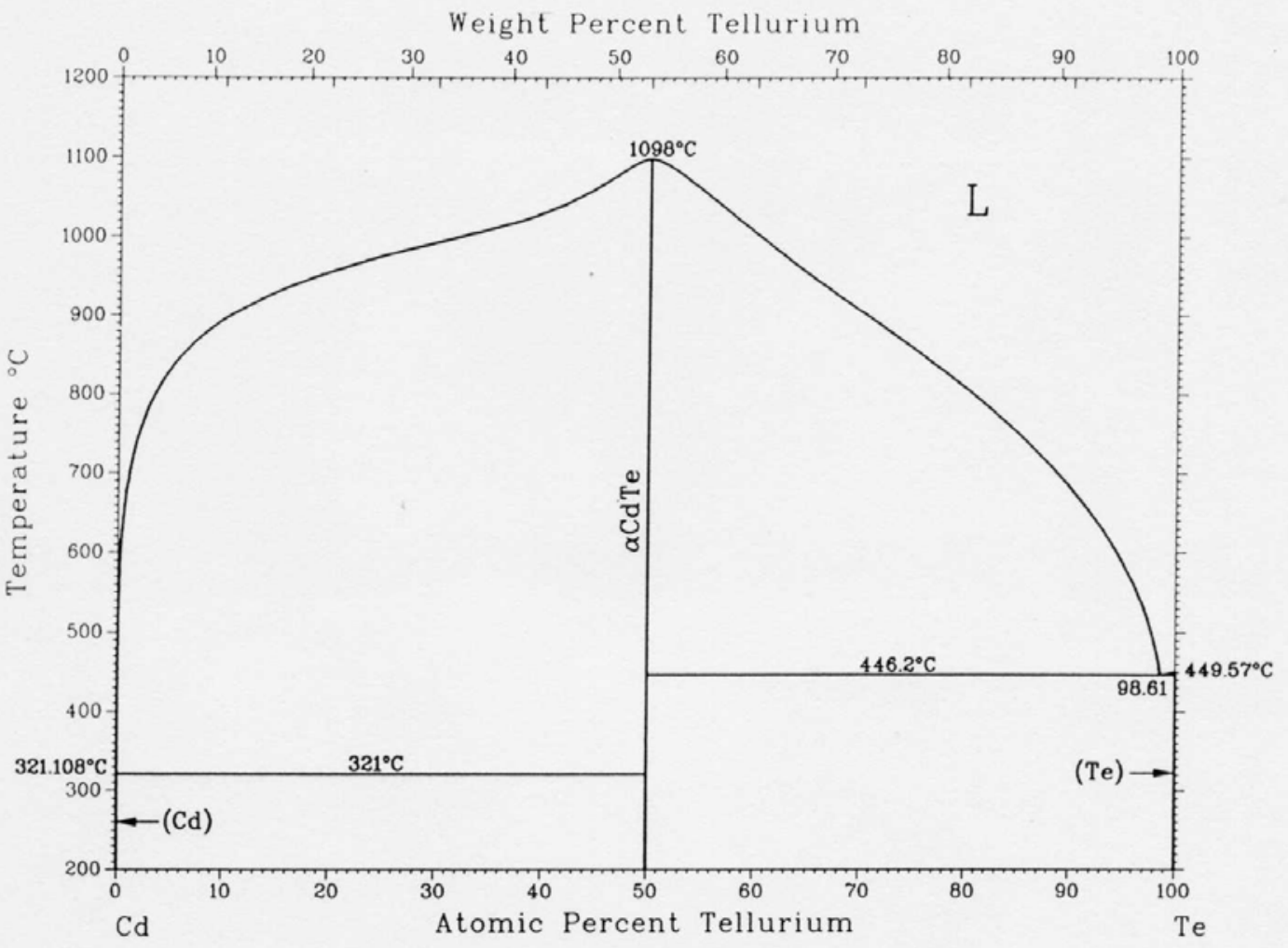

FIG. 9. Diagramme des phases du système cadmium-tellure, montrant l'existence d'un composé CdTe purement stæchiométrique [24].

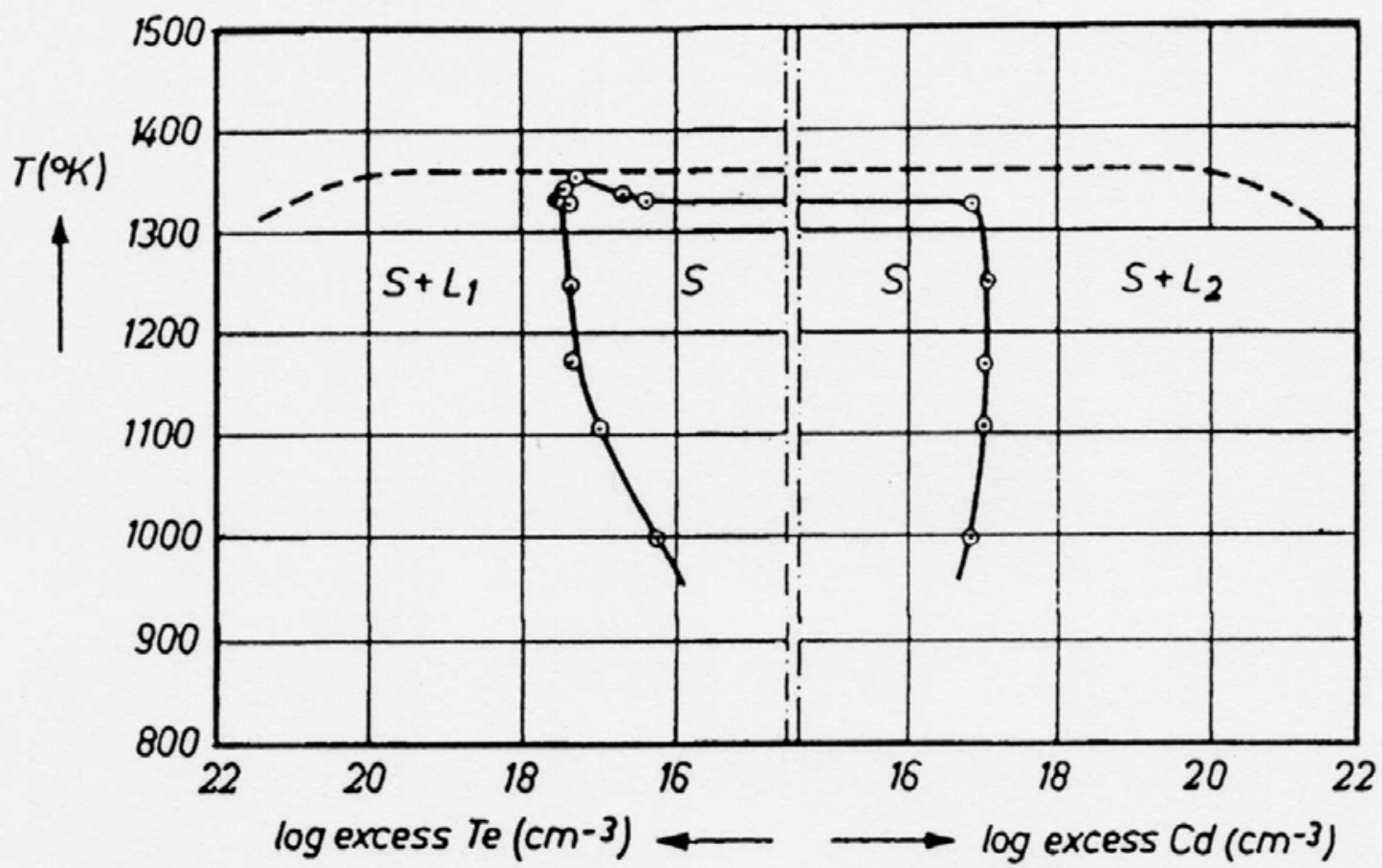

FIG. 10. Détail du diagramme des phases du système cadmium-tellure à haute température et au voisinage de la composition stœchiométrique [25]. 


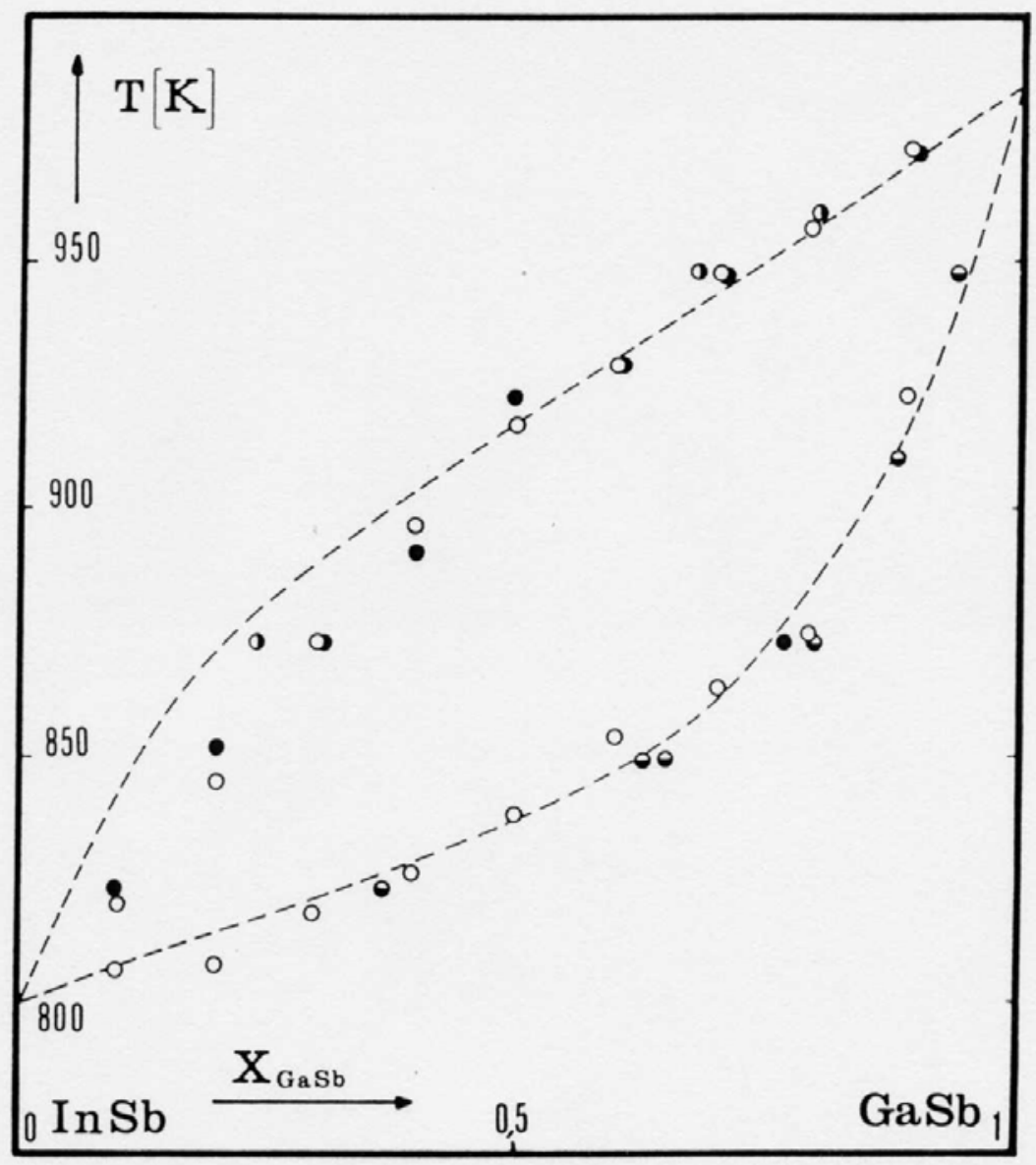

FIG. 11. Diagramme des phases du système quasi-binaire GaSb-InSb montrant un exemple de miscibilité complète avec solution solide couvrant tout le domaine de composition [26].

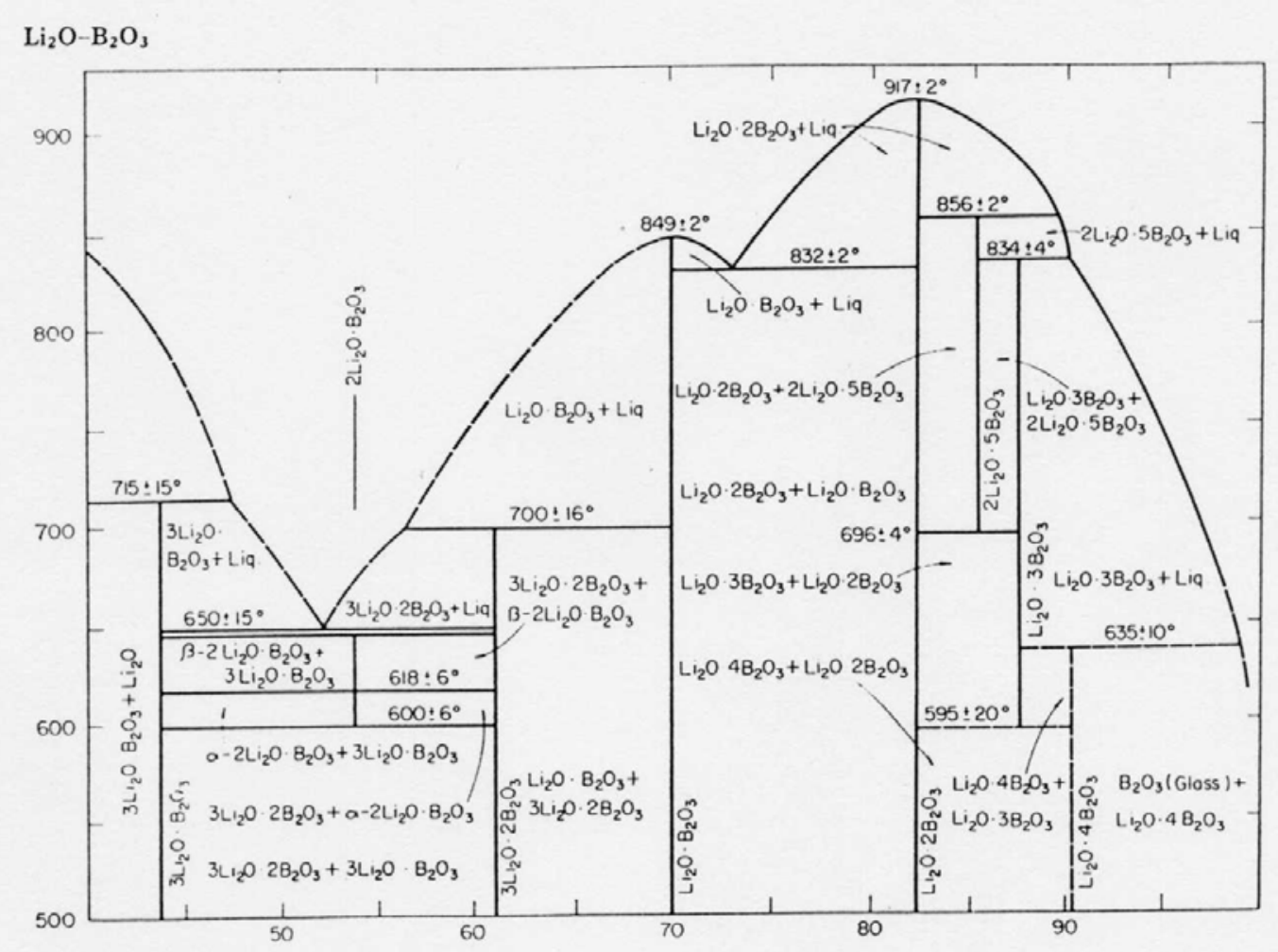

FIG. 12. Diagramme des phases du système quasi-binaire $\mathrm{Li}_{2} \mathrm{O}-\mathrm{B}_{2} \mathrm{O}_{3}$ permettant d'évaluer la faisabilité de mise en oeuvre des techniques de croissance pour chaque composé défini [27]. 
Un autre objectif fréquent dans la pratique est d'obtenir un matériau comprenant un constituant en très faible proportion permettant de conférer à l'alliage les propriétés souhaitées, ce dernier étant alors désigné sous le terme de dopant. On peut citer par exemple le cas du grenat d'yttrium et d'aluminium dopé au néodyme $\mathrm{Y}_{3-\mathrm{x}} \mathrm{Nd}_{\mathrm{x}} \mathrm{Al}_{5} \mathrm{O}_{12}$ (YAG:Nd) pour lequel la connaissance de tous les recoins du diagramme $(\mathrm{Y}, \mathrm{Nd}, \mathrm{Al}, \mathrm{O})$ n'est heureusement pas nécessaire. $\mathrm{Vu}$ l'importance de cette configuration de matériau dopé, il est utile d'y détailler les mécanismes d'incorporation du soluté. Intéressons-nous pour ce faire au voisinage du composé GaSb sur le diagramme des phases de la figure 11, et considérons un alliage à une teneur $C_{0}=5 \%$ atomique en InSb refroidi à partir de la phase liquide.

La concentration en InSb dans le cristal formé étant de l'ordre de $1 \%$, la solution va s'enrichir en InSb, les points représentatifs du solide et du liquide évoluant le long des courbes solidus et liquidus. Pour les matériaux dopés, ces courbes sont souvent assimilables à des droites, de pentes respectives $m_{\mathrm{S}}$ et $m_{\mathrm{L}}$. Le coefficient de partage $k$ de l'alliage, défini comme le rapport des concentrations au front de croissance $C_{\mathrm{S}}{ }^{\mathrm{I}} / C_{\mathrm{L}}^{\mathrm{I}}$ peut alors être considéré constant :

$$
\mathrm{k}=\mathrm{C}_{\mathrm{S}}{ }^{\mathrm{I}} / \mathrm{C}_{\mathrm{L}}{ }^{\mathrm{I}}=m_{\mathrm{L}} / m_{\mathrm{S}}
$$

De façon générale, un coefficient de partage proche de l'unité permet de limiter la ségrégation chimique dans le matériau solidifié, puisque dans le cas limite $k=1$ un cristal homogène pourra être obtenu à partir d'un bain liquide où les mouvements de convection (voir section $\underline{4.2}$ ) auront fait disparaître d'éventuels gradients de concentration créés lors de la phase de fusion. Ce cas idéal n'est que très rarement rencontré dans la pratique, et nous verrons en section $\underline{4.3}$ que les phénomènes de ségrégation sont d'autant plus marqués que k est différent de l'unité. À ce sujet, signalons que les situations où $k<<1$, i.e. celles où l'incorporation en phase solide du soluté est quasi-impossible, sont assez fréquentes dans la pratique, mais il existe également des systèmes à $k>1$ où le solide formé contient plus de soluté que le liquide au front de croissance.

Un autre cas favorable du point de vue de la ségrégation chimique se rencontre quand le système parvient à un régime stationnaire où la concentration du solide formé est égale à la concentration nominale de l'alliage. Cela dit, nous allons voir dans la suite que les compositions au front de croissance sont fonction des mécanismes de transport de soluté, et que ce régime stationnaire n'est accessible que si le transfert de masse en phase liquide est purement diffusif. La température de l'interface solide-liquide ne peut donc pas être connue a priori, la thermodynamique des systèmes d'équilibre nous enseigne seulement qu'elle sera typiquement comprise entre $T_{\mathrm{L}}\left(C_{0}\right)$ et $T_{\mathrm{S}}\left(C_{0}\right)$, où $T_{\mathrm{L}}\left(C_{0}\right)$ et $T_{\mathrm{S}}\left(C_{0}\right)$ représentent respectivement les températures de liquidus et de solidus à la composition $C_{0}$. La différence $\Delta T^{\mathrm{M}}$ entre $T_{\mathrm{S}}\left(C_{0}\right)$ et $T_{\mathrm{L}}\left(C_{0}\right)$ peut s'exprimer par :

$$
\Delta T^{\mathrm{M}}=T_{\mathrm{S}}\left(C_{0}\right)-T_{\mathrm{L}}\left(C_{0}\right)=\left(m_{\mathrm{S}}-m_{\mathrm{L}}\right) C_{0}=m_{\mathrm{L}}[(1-k) / k] C_{0} .
$$

Cette différence induit une incertitude sur la position du front de croissance dans le dispositif expérimental, qui sera d'autant plus marquée que le gradient de température sera faible. Pour fixer les idées, reprenons notre exemple du système GaSb-InSb à une concentration $C_{0}=5 \%$ atomique en InSb. En utilisant les valeurs des pentes des liquidus et solidus estimées à partir du diagramme des phases, $m_{\mathrm{S}}=-6,5 \mathrm{~K} / \% \mathrm{InSb}$ et $m_{\mathrm{L}}=-1,3 \mathrm{~K} / \% \mathrm{InSb}$, la formule précédente donne une valeur de plus de $30 \mathrm{~K}$ pour $\Delta T^{\mathrm{M}}$. Avec une valeur typique du gradient thermique $G_{\mathrm{T}}=10 \mathrm{~K} / \mathrm{cm}$, on voit que l'incertitude est supérieure à $3 \mathrm{~cm}$, ce qui est considérable. Une valeur plus élevée de $G_{\mathrm{T}}$ permet évidemment de réduire cette incertitude, mais nous avons vu en section $\underline{3}$ que les problèmes de qualité structurale limitent les possibilités en matière de choix du gradient thermique.

Pour conclure, il importe de bien garder à l'esprit que la connaissance du diagramme des phases de l'alliage à élaborer est une nécessité pour le contrôle du procédé de croissance. En l'absence de diagramme publié dans la littérature, l'expérimentateur se doit de procéder empiriquement pour délimiter les domaines de stabilité des diverses phases en présence dans un espace températureconcentration. Signalons enfin que le développement des codes de calcul thermodynamiques a conduit à une meilleure connaissance des diagrammes des phases dans certains systèmes complexes. Il reste que le manque, l'ancienneté ou l'imprécision des données rend la tâche de l'expérimentateur souvent difficile. 


\subsection{Mécanismes de transport}

Du point de vue pratique, l'objectif de l'expérimentateur est bien évidemment le contrôle de la composition du matériau élaboré dans les conditions d'utilisation, généralement à basse température. Nous allons cependant voir que, dans la mesure où les mécanismes de diffusion en phase solide sont très limités, l'incorporation du soluté dans le cristal est gouvernée par son transport en phase liquide et en particulier par la convection naturelle. Rappelons à ce sujet que l'étude de la thermique des dispositifs n'entre pas dans notre propos (voir par exemple [2] pour une synthèse globale), mais que le processus de croissance se déroule généralement en présence de gradients de température. Nous ne considérerons ici que le cas d'un alliage binaire, et supposerons de plus que les densités massiques des phases solide et liquide sont égales, le lecteur pouvant se reporter à la référence [28] pour une présentation plus complète.

Nous ne nous intéresserons plus à partir de maintenant au cas trivial où le coefficient de partage $\mathrm{k}$ est égal à l'unité, puisque ce cas ne pose pas de difficultés en matière de ségrégation. Pour les systèmes à coefficient de partage $k<1$, le soluté non incorporé à l'interface doit diffuser à partir du front de croissance, et la condition locale de conservation de la masse peut s'écrire sous la forme :

$$
D_{\mathrm{S}}\left(\partial C_{\mathrm{S}} / \partial n\right)_{\mathrm{I}}-D_{\mathrm{L}}\left(\partial C_{\mathrm{L}} / \partial n\right)_{\mathrm{I}}=\mathbf{V}_{\mathbf{I}} \mathbf{n}\left(C_{\mathrm{L}}{ }^{\mathrm{I}}-C_{\mathrm{S}}{ }^{\mathrm{I}}\right),
$$

où les coefficients de diffusion dans les phases solide et liquide sont respectivement notés $D_{\mathrm{S}}$ et $D_{\mathrm{L}}, \mathbf{V}_{\mathbf{I}}$ et $\mathbf{n}$ représentant la vitesse de solidification imposée par l'expérimentateur et la normale au front de croissance pointant vers le liquide. Du point de vue du formalisme, il est préférable d'exprimer les concentrations en fractions massiques pour faire le lien avec les équations de la mécanique des fluides que nous allons bientôt présenter. Cela posé, on peut montrer qu'il est possible d'utiliser également les fractions molaires dans le cas des alliages dilués [28]. Pour les systèmes à coefficient de partage $k>1$, le soluté doit diffuser vers l'interface, et le sens des gradients est inversé, mais la condition de conservation locale, i.e. au front de croissance, donnée par l'équation (21) reste inchangée. Rappelons enfin que nous avons vu en section 4.1 que le diagramme des phases pouvait être utilisé pour exprimer une relation entre $C_{\mathrm{L}}{ }^{\mathrm{I}}$ et $C_{\mathrm{S}}{ }^{\mathrm{I}}$ sous la forme $C_{\mathrm{S}}{ }^{\mathrm{I}}=f\left(C_{\mathrm{L}}{ }^{\mathrm{I}}\right)$.

Une équation globale de conservation de la masse de soluté dans chacune des phases peut également être écrite sous la forme générale :

$$
\partial\left(\rho_{\xi} C\right) / \partial t+\nabla \cdot \mathbf{J}_{\xi}^{\mathbf{M}}=0
$$

où $\rho_{\xi}$ et $\mathbf{J}_{\xi}^{\mathbf{M}}(\xi=\mathrm{L}$ ou $\mathrm{S})$ représentent respectivement la masse volumique et le flux massique total dans la phase considérée. Dans le solide, le transport se réduit au premier ordre à un flux diffusif, $\mathbf{J}_{\mathbf{S}} \mathbf{M}=-\rho_{\mathrm{S}} D_{\mathrm{S}} \nabla C_{\mathrm{S}}$, mais les valeurs du coefficient de diffusion sont extrêmement faibles, typiquement de l'ordre de $10^{-15}$ à $10^{-11} \mathrm{~m}^{2} \mathrm{~s}^{-1}$ au voisinage du point de fusion. En supposant un processus de solidification tel que le système soit maintenu à des températures élevées sur une durée de l'ordre de quelques heures, la longueur caractéristique de diffusion $l=\left(D_{\mathrm{S}} t\right)^{1 / 2}$ est au maximum de l'ordre de la centaine de micromètres. À des échelles plus importantes, en particulier celle de la dimension macroscopique du cristal, le soluté ne peut se redistribuer dans la phase solide. Nous supposerons donc dans la suite que l'étude du problème de transport peut être restreint à la phase liquide, où le flux massique total s'exprime sous la forme de la somme d'un terme de diffusion et d'un terme de convection :

$$
\mathbf{J}_{\mathbf{L}}^{\mathbf{M}}=-\rho_{\mathrm{L}} D_{\mathrm{L}} \nabla C_{\mathrm{L}}+\rho_{\mathrm{L}} \mathbf{V} C_{\mathrm{L}} .
$$

La vitesse du fluide $\mathbf{V}$ est gouvernée par les équations de la mécanique des fluides, connues sous le nom d'équations de Navier-Stokes, que nous écrirons sous la forme :

$$
\rho_{\mathrm{L}}[\partial \mathbf{V} / \partial t+(\mathbf{V} . \nabla) \mathbf{V}]=-\nabla P+\eta \nabla^{2} \mathbf{V}+\mathbf{F}_{\text {ext }} .
$$

L'équation ci-dessus est représentative d'un fluide newtonien, hypothèse communément admise pour les fluides « simples » rencontrés en cristallogenèse. Les symboles $P$ et $\eta$ représentent respectivement la pression et la viscosité dynamique du matériau, l'opérateur nabla de différentiation étant noté $\nabla$. Le terme $\mathbf{F}_{\text {ext }}$ mesure la densité volumique de forces extérieures s'appliquant au système. D'un point de vue général, le transport convectif est souvent source de ségrégation solutale dans les expériences de cristallogenèse (voir section $\underline{4.3}$ ) et quelques mots sont nécessaires pour discuter les sources principales de mouvement et les moyens de contrôle associés. 
En prenant le rotationnel de l'équation (24), on constate qu'une condition nécessaire pour que le fluide soit au repos est que $\nabla \times \mathbf{F}_{\text {ext }}$ soit partout égal à zéro. Cette condition est très difficile à remplir dans la pratique. Considérons en effet une source "naturelle» de convection, à savoir le champ de pesanteur. Les variations de température et de concentration par rapport à des valeurs de référence $T_{0}$ et $C_{0}$ font que $\mathbf{F}_{\text {ext }}$ s'exprime par :

$$
\mathbf{F}_{\text {ext }}=\rho_{\mathrm{L}}\left[1-\beta_{\mathrm{T}}\left(T-T_{0}\right)+\beta_{\mathrm{C}}\left(C-C_{0}\right)\right] \mathbf{g} .
$$

Le rotationnel de l'expression précédente ne peut être nul que si les gradients de densité sont partout alignés avec le vecteur gravité. Pour les alliages dilués où le terme thermique est prépondérant, $\nabla \times \mathbf{F}_{\text {ext }}=\mathbf{0}$ impose l'absence de gradients de température horizontaux, condition irréalisable dans la pratique. Pour les alliages concentrés, les termes thermique et solutal peuvent se compenser dans une certaine mesure, mais on peut se risquer à affirmer que dans une expérience de cristallogenèse typique, le fluide sera toujours soumis à des mouvements de convection naturelle.

Une façon de contourner le problème est de recourir à l'environnement inertiel de vaisseaux spatiaux orbitant autour de la terre, où le champ de pesanteur est très fortement réduit, $\mathbf{g} \approx 0$. De nombreux résultats intéressants en terme de mise en évidence de mécanismes de transport ont ainsi été obtenus. Cela dit, si l'environnement microgravité est bien adapté aux expériences de démonstration, la production en masse de nouveaux matériaux n'y est pas envisageable dans un futur raisonnable.

Pour un liquide présentant des surfaces sans contact avec une paroi solide, une autre source de convection naturelle est la variation de sa tension de surface $\sigma$ avec la température et la concentration. En effet, la condition d'équilibre mécanique de l'interface liquide-gaz peut s'exprimer sous la forme :

$$
-\eta \partial U / \partial n=\partial \sigma / \partial u=(\partial \sigma / \partial T)_{0} \partial T / \partial u+(\partial \sigma / \partial C)_{0} \partial C / \partial u
$$

où $U$ représente la composante du vecteur vitesse $\mathbf{V}$ le long d'une direction $\mathbf{u}$ tangente à la surface libre. Ce mécanisme est désigné dans la littérature sous le nom d'effet Marangoni [29]. Son effet est loin d'être négligeable, puisque l'équation précédente montre qu'une force motrice localisée à l'interface va induire des mouvements dans la direction $\mathbf{n}$ normale à la surface libre, i.e. en profondeur dans le fluide.

Dans une optique de contrôle des mouvements convectifs en phase liquide, une possibilité intéressante est d'utiliser des champs magnétiques, le mécanisme physique étant dans ce cas la prise en compte de la force de Laplace : $\mathbf{F}_{\text {ext }}=\mathbf{j}$ x $\mathbf{B}$ dans les équations de Navier-Stokes. On montre que selon la nature statique ou dynamique du champ magnétique, on peut freiner ou accélérer les mouvements du fluide [30]. Un autre moyen de contrôle de l'écoulement est d'imposer une rotation au cristal et/ou au creuset : cette convection, dite forcée, est en particulier très utilisée en configuration Czochralski.

Pour terminer cette brève introduction aux mécanismes de transport, un certain nombre d'éléments peuvent être signalés. Pour spécifier le problème mécanique, il est nécessaire d'associer à l'équation de la dynamique des fluides une équation de conservation de la masse, dite de continuité, qui peut se mettre sous la forme :

$$
\partial \rho_{\mathrm{L}} / \partial t+\nabla \cdot\left(\rho_{\mathrm{L}} \mathbf{V}\right)=0
$$

Pour les fluides isochores (appelés par abus de langage incompressibles) habituellement rencontrés dans les expériences de cristallogenèse en phase liquide, l'expression précédente se réduit à $\nabla \cdot \mathbf{V}=0$. Toujours sur le problème de mécanique, si les variations relatives de densité sont suffisamment modestes, on suppose que la prise en compte de l'effet des variations de température et de concentration peut être limitée au terme de pesanteur, la densité étant supposée constante dans les autres termes de l'équation de Navier-Stokes.

Concernant le champ de concentration, nous avons discuté en détail l'équation (21) qui traduit la conservation locale du soluté à l'interface de croissance, mais d'autres conditions aux limites sont nécessaires pour spécifier le problème. On suppose généralement le flux de masse $\mathbf{J}_{\mathbf{L}}{ }^{\mathbf{M}}$.n imposé sur les frontières de la phase liquide, le cas particulier des parois imperméables se traduisant par $\mathbf{J}_{\mathbf{L}}{ }^{\mathbf{M}} \cdot \mathbf{n}=0$. Par ailleurs, la formulation présentée peut être facilement étendue aux systèmes polyconstitués, sous réserve que les équations (21)-(23) puissent être écrites pour chaque soluté indépendamment. Signalons enfin qu'il est parfois intéressant de traiter le problème dans un repère mobile avec la vitesse $\mathbf{V}_{\mathbf{I}}$ du front de croissance car il est alors possible d'atteindre un régime 
quasi-permanent comme nous le verrons en section 4.3.2. Dans ce repère mobile, on doit ajouter un terme $-\rho_{\mathrm{L}} \mathbf{V}_{\mathrm{I}} C_{\mathrm{L}}$ au flux de masse total, et l'équation de conservation (22) peut se mettre sous la forme :

$$
\partial C_{\mathrm{L}} / \partial t+(\mathbf{V} \cdot \nabla) C_{\mathrm{L}}=D_{\mathrm{L}} \nabla^{2} C_{\mathrm{L}}+\left(\mathbf{V}_{\mathbf{I}} \cdot \nabla\right) C_{\mathrm{L}}
$$

Une fois le problème bien posé du point de vue mathématique, il est souvent tellement complexe que sa résolution n'en est pas pour autant évidente. Ce point peut être illustré en considérant les diverses forces motrices pour l'hydrodynamique du procédé Czochralski. Tout d'abord, la présence de zones chaudes au voisinage des parois latérales du creuset va induire des mouvements de convection naturelle dont l'effet se fera sentir jusqu'au voisinage du cristal en cours de croissance. L'existence de surfaces libres à proximité du point triple solide-liquide-atmosphère est pour sa part source de mouvements de convection Marangoni. Enfin, la rotation imposée au cristal et éventuellement au creuset induit un écoulement forcé dans le bain. L'interaction entre ces diverses sources convectives fait que la résolution des équations de Navier-Stokes est d'une grande complexité.

Sans vouloir rentrer dans les détails des problèmes de thermique, on peut mentionner que le calcul du champ de température dans les dispositifs expérimentaux est également très complexe, puisque l'estimation des flux radiatifs à haute température nécessite l'identification des facteurs de vue dans l'enceinte. Ce problème de rayonnement est rendu encore plus délicat par le fait que les matériaux de structure évoluent au cours de la vie du dispositif, avec pour conséquence des modifications de leurs propriétés d'émissivité. Le calcul des flux radiatifs dans l'échantillon, souvent nécessaire dans les matériaux transparents comme les oxydes, n'est pas non plus évident. Signalons enfin que les variations de la masse volumique et de la tension de surface avec la température et la concentration induisent un couplage entre les champs hydrodynamique, thermique et solutal.

Ce couplage est bien évidemment source de complexité, même si des simplifications sont parfois possibles. Par exemple, en configuration Bridgman vertical, les mouvements du fluide sont généralement assez lents pour que le transfert de chaleur soit considéré principalement diffusif. Si tel est le cas, le problème thermique peut être découplé et résolu séparément. Cela dit, la validité de cette hypothèse doit toujours être vérifiée a posteriori en calculant un nombre de Peclet thermique par la formule $P e_{\mathrm{T}}=V H / \alpha_{\mathrm{L}}$, où $H$ et $V$ représentent respectivement une vitesse et une dimension caractéristique de l'écoulement, $\alpha_{\mathrm{L}}$ mesurant la diffusivité thermique du matériau.

Une autre simplification possible dans le cas des alliages dilués est celle qui consiste à négliger les variations en concentration des densités et tensions de surface, ce qui permet de découpler l'équation de Navier-Stokes de celle du transport solutal. Il faut pour ce faire s'assurer a posteriori que le rapport des vitesses d'écoulement induites par les gradients de composition d'une part et de température d'autre part reste très petit devant l'unité. Un autre point à signaler est que dans les systèmes concentrés, les paramètres thermodynamiques $\left(k, m_{\mathrm{L}}\right)$ et cinétiques $(\eta, D)$ dépendent de la concentration, ce qui complique encore le problème.

On comprend donc que, même si certaines simplifications sont parfois possibles, la résolution des équations de la thermique, de la mécanique des fluides et du transport solutal nécessite en général le recours aux méthodes numériques. L'évolution des moyens de calcul au cours des deux dernières décennies a permis d'enregistrer des progrès considérables, et on peut penser que cette dynamique va permettre d'affiner encore les hypothèses des modèles. Il reste que le manque de données thermophysiques dans la littérature est un obstacle sérieux à la fiabilité des calculs. On peut donc affirmer que le développement des méthodes numériques passe également par la réalisation de programmes de mesure de données expérimentales, notamment en matière de coefficients de transport.

\subsection{Lois de ségrégation}

Notre objectif dans cette section est de montrer qu'il est possible de quantifier les variations de concentration dans les matériaux élaborés. Nous supposerons pour ce faire le problème solutal monodimensionnel, i.e. que la composition dans les cristaux ne dépend que de l'abscisse mesurée dans la direction de croissance, notée ici $Z$, une étude des phénomènes de ségrégation radiale dépassant largement le cadre de ce texte. Une autre hypothèse sera de considérer que le coefficient de partage $\mathrm{k}$ est indépendant de la concentration. Le bain fondu sera par ailleurs supposé homogène avant la solidification, la composition nominale de l'alliage étant notée $C_{0}$. 


\subsubsection{Mélange complet dans le bain}

Une hypothèse simple permettant de calculer le profil de concentration le long d'un barreau solidifié directionnellement est de supposer que la composition du bain liquide est à chaque instant homogène. Remarquons que cette hypothèse n'est pas stricto sensu compatible avec l'équation (21) représentant la conservation du soluté au front de croissance, sauf à considérer une vitesse d'interface $V_{\mathrm{I}}$ nulle ou un coefficient de diffusion $D_{\mathrm{L}}$ infini. Nous verrons cependant en section $\underline{4.3 .3}$ que la présence de mouvements convectifs intenses dans le bain fondu, une situation assez fréquente dans la pratique, permet une homogénéisation quasi-parfaite de la composition. Quoi qu'il en soit le calcul de la concentration $C_{\mathrm{S}}(Z)=k C_{\mathrm{L}}(Z)$ à la côte $Z$ d'un barreau de longueur $L$ peut se faire en écrivant un bilan de conservation. En effet, la quantité de soluté incorporée dans le cristal doit être égale à la variation de la masse de soluté présente en phase liquide $Q$, cette dernière étant proportionnelle à $(L-Z) C_{\mathrm{L}}(Z)$. Nous ne détaillerons pas ici le calcul, le lecteur pouvant par exemple se reporter à la référence [28] pour une présentation plus complète. Signalons seulement qu'une intégration de la relation $\mathrm{d} Q=-k C_{\mathrm{L}}(Z)$ à partir de la condition initiale $C_{\mathrm{L}}=C_{0}$ en $Z=0$ permet d'accéder au profil de concentration :

$$
C_{\mathrm{S}}(Z)=k C_{\mathrm{L}}(Z)=k C_{0}\left(1-f_{\mathrm{S}}\right)^{(k-1)},
$$

où la fraction solidifiée $f_{\mathrm{S}}$ est définie par $f_{\mathrm{S}}=Z / L$. L'équation précédente est connue dans la littérature sous le nom d'équation de Scheil-Gulliver. Les profils correspondants sont représentés en figure $\underline{13}$ pour différentes valeurs de $k$ [31]. On constate que les variations de concentration le long du barreau peuvent être très importantes pour les coefficients de partage significativement différents de l'unité. Cela posé, comme nous l'avons déjà signalé pour le problème des contraintes de croissance, les variations acceptables pour une application donnée doivent être quantifiées au cas par cas par les utilisateurs. Il importe par ailleurs de remarquer que l'équation (29) ne peut rester valable jusqu'à la fin de la solidification $\left(f_{\mathrm{S}}=1\right)$ pour les valeurs de $k<1$, puisque la concentration prédite devient infinie. Dans la pratique, plusieurs mécanismes peuvent être activés :

- $\quad$ à gradients de température et vitesse de croissance donnés, l'interface solide-liquide subit une instabilité morphologique quand la concentration dépasse le seuil de Mullins-Sekerka [32], avec formation de cellules ou de dendrites. Cette transition structurale, sur laquelle nous reviendrons en section $\underline{4.4}$, fait que le problème ne peut plus être considéré mono-dimensionnel.

- $\quad$ comme nous l'avons vu en section 4.1, la solubilité du soluté en phase solide est généralement limitée. Une structure eutectique ou péritectique va donc se former quand la composition dépasse un certain seuil, et il devient ici également nécessaire de tenir compte du transport de masse dans les directions normales à celle de la croissance.

- $\quad$ même dans les systèmes à miscibilité totale (Fig. 11), le coefficient de partage ne peut rester constant quand la concentration devient trop élevée, et il n'est plus possible de réaliser simplement l'intégration de la relation de conservation.

Pour conclure, signalons qu'il est assez remarquable que les profils de concentration observés expérimentalement soient souvent proches de ceux prédits par l'équation de Scheil-Gulliver, alors que nous avons vu que l'hypothèse de bain homogène était discutable d'un point de vue théorique. Nous reviendrons sur ce point en section $\underline{4.3 .3}$ pour montrer que ce résultat a priori surprenant est en fait facilement explicable.

\subsubsection{Transport solutal diffusif}

Une autre hypothèse permettant de quantifier le profil de concentration dans les cristaux élaborés est de supposer que le transport solutal est purement diffusif, ou, en d'autres termes, que la vitesse de convection solution des équations de Navier-Stokes est partout nulle. Dans le repère mobile à la vitesse $\mathbf{V}_{\mathbf{I}}$ du front de croissance, l'équation régissant la conservation de la masse dans la phase liquide peut se mettre sous la forme :

$$
\partial C_{\mathrm{L}} / \partial t=D_{\mathrm{L}} \partial^{2} C_{\mathrm{L}} / \partial Z_{\mathrm{L}}^{2}+V_{\mathrm{I}} \partial C_{\mathrm{L}} / \partial Z_{\mathrm{L}}
$$




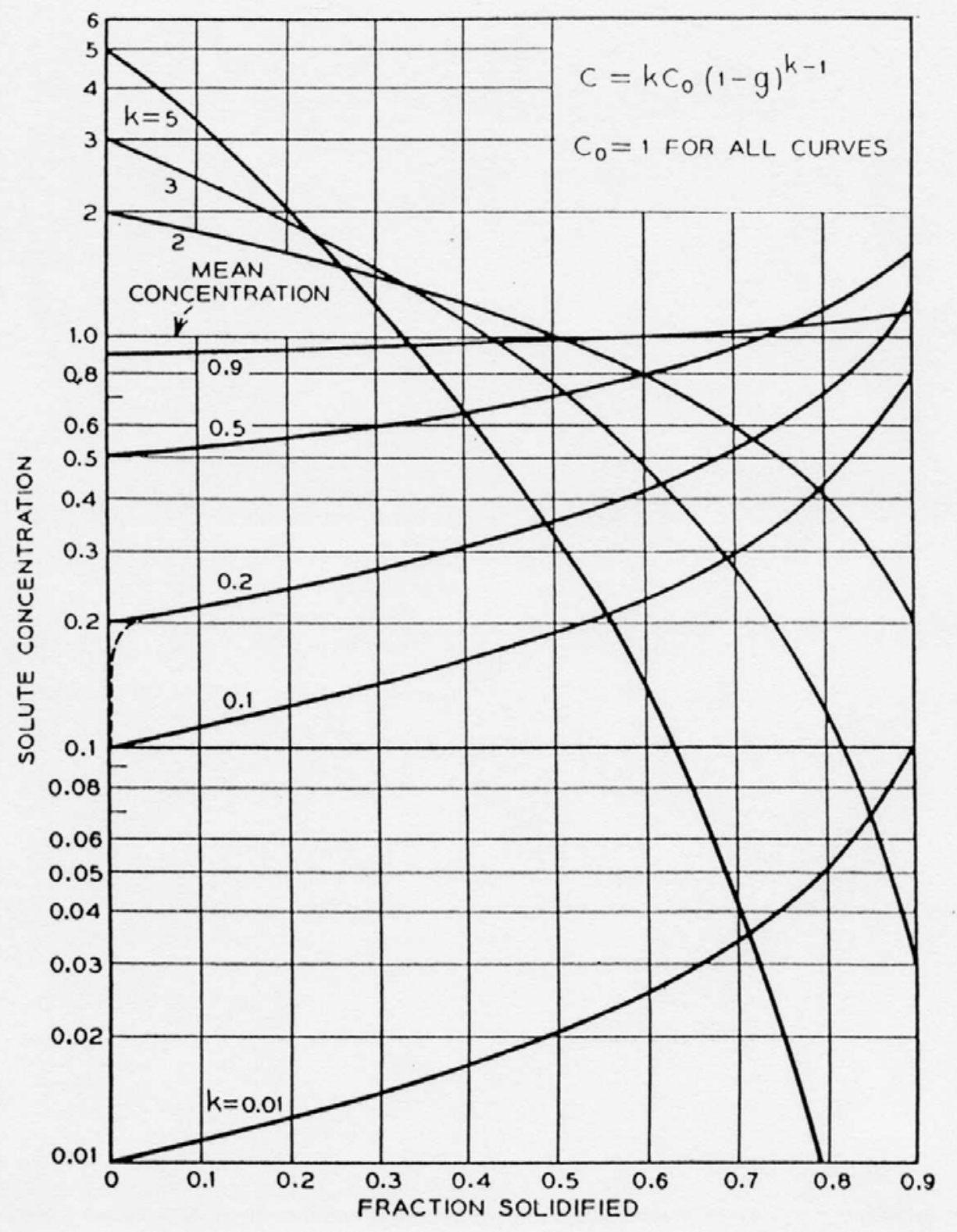

FIG. 13. Représentation des profils de concentration le long du cristal dans l'hypothèse de mélange complet dans le bain (cf. équation (29), loi de Scheil), pour diverses valeurs du coefficient de partage $k$ [31].

où la distance au front de croissance est notée $Z_{\mathrm{L}}$. Cette équation différentielle ne peut être résolue sans conditions aux limites. Il importe de bien comprendre que l'équation (21) régissant la conservation du soluté à l'interface de croissance est indépendante du mode de transport dans le bain. Nous utiliserons dans la suite une formulation bien adaptée aux problèmes monodimensionnels dans les alliages dilués :

$$
-D_{\mathrm{L}}\left(\partial C_{\mathrm{L}} / \partial Z_{\mathrm{L}}\right)_{\mathrm{I}}=V_{\mathrm{I}}(1-k) C_{\mathrm{L}}^{\mathrm{I}} .
$$

Il nous faut également spécifier une condition à l'autre extrémité du domaine liquide, supposé ici infini pour des raisons de commodité mathématique. En prenant $C_{\mathrm{L}} \rightarrow C_{0}$ quand $Z_{\mathrm{L}} \rightarrow \infty$, on trouve facilement que le système admet une solution quasi stationnaire $\left(\partial C_{\mathrm{L}} / \partial t=0\right)$ de la forme :

$$
C_{\mathrm{L}}\left(Z_{\mathrm{L}}\right)=C_{0}\left\{1+[(1-k) / k] \exp \left(-V_{\mathrm{I}} Z_{\mathrm{L}} / D_{\mathrm{L}}\right)\right\} .
$$

La composition dans le liquide à l'interface $\left(Z_{\mathrm{L}}=0\right)$ étant égale à $C_{0} / k$, cette solution montre qu'il est possible d'atteindre un régime optimal du point de vue de la ségrégation où la composition dans le solide formé est égale à la composition nominale de l'alliage, $C_{\mathrm{S}}=C_{0}$. Il est intéressant par ailleurs de remarquer que les variations de concentration dans le liquide se font dans une couche limite d'épaisseur caractéristique $\delta=D_{\mathrm{L}} / V_{\mathrm{I}}$ à l'avant du front de solidification. 
L'équation (32) permet de préciser les hypothèses de départ: tout d'abord, l'infini de la condition aux limites doit être mesuré à l'échelle de la couche limite elle-même. Dans la pratique, vu sa structure exponentielle, la solution sera correcte dès que la dimension du bain fondu dépassera quelques $D_{\mathrm{L}} / V_{\mathrm{I}}$. Plus important, il n'est en fait pas nécessaire pour la validité de l'équation (2) que la vitesse de convection soit nulle partout dans le bain, une condition suffisante est que le transport convectif soit négligeable à l'échelle de la couche limite. Le front de croissance étant une frontière où la vitesse de convection doit s'annuler (hypothèse de non glissement sur les surfaces solides), la validité de cette condition dépend fortement de la vitesse de solidification $V_{\mathrm{I}}$ imposée par l'utilisateur.

En effet, pour une valeur typique du coefficient de diffusion en phase liquide $D_{\mathrm{L}}=5.10^{-9} \mathrm{~m}^{2} \cdot \mathrm{s}^{-1}$, une vitesse rapide $V_{\mathrm{I}}=1 \mathrm{~mm} \cdot \mathrm{s}^{-1}$ conduit à $\delta=D_{\mathrm{L}} / V_{\mathrm{I}}=5 \mu \mathrm{m}$. Dans ce cas, la couche limite étant très proche de la frontière de non glissement, la convection ne pourra que difficilement agir sur le transport solutal. En revanche, pour une vitesse relativement lente $V_{\mathrm{I}}=1 \mu \mathrm{m} / \mathrm{s}$, on trouve $\delta=D_{\mathrm{L}} / V_{\mathrm{I}}=5 \mathrm{~mm}$. À cette échelle, l'hypothèse de régime diffusif devient beaucoup plus discutable dans la mesure où la convection est moins contrainte par la proximité du front de croissance.

Travailler à des vitesses de solidification élevées permet donc en principe de limiter la ségrégation, mais le mécanisme de déstabilisation morphologique déjà évoqué en section 4.3.1 fait que $V_{\mathrm{I}}$ ne doit pas dépasser le seuil de Mullins-Sekerka. Un autre point à mentionner est qu'une phase transitoire, de dimension caractéristique $D_{\mathrm{L}} / k V_{\mathrm{I}}$ est nécessaire avant d'atteindre le régime quasipermanent. En prenant $D_{\mathrm{L}}=5 \cdot 10^{-9} \mathrm{~m}^{2} \cdot \mathrm{s}^{-1}, V_{\mathrm{I}}=1 \mu \mathrm{m} \cdot \mathrm{s}^{-1}$ et $k=0,2$, il vient $D_{\mathrm{L}} / k V_{\mathrm{I}}=1 \mathrm{~cm}$. Pour des valeurs plus faibles du coefficient de partage, cette phase transitoire peut s'étendre sur toute la longueur du cristal. Ces limitations font que le régime de croissance à composition homogène est malheureusement difficile à atteindre dans la pratique.

\subsubsection{Coefficient de partage effectif}

Quel que soit le mode de transport dans le bain, l'équation (31) montre qu'il existe une couche limite solutale enrichie (pour $k<1$ ) ou appauvrie (pour $k>1$ ) à l'avant du front de solidification, et cette idée a été confirmée par un bon nombre de résultats expérimentaux ou de simulation numérique. L'effet de la convection est globalement de réduire l'épaisseur $\delta$ de cette couche limite par rapport à sa valeur $D_{\mathrm{L}} / V_{\mathrm{I}}$ en régime diffusif. Du point de vue mathématique, $\delta$ peut être définie à partir de la relation suivante :

$$
\delta=-\left(C_{\mathrm{L}}^{\mathrm{I}}-C_{\mathrm{L}}^{\infty}\right) /\left(\partial C_{\mathrm{L}} / \partial Z_{\mathrm{L}}\right)_{\mathrm{I}},
$$

où $C_{\mathrm{L}}{ }^{\infty}$ représente la concentration dans le bain fondu loin de l'interface, cet infini devant bien sûr être encore mesuré à l'échelle de la couche limite elle-même. En régime de croissance diffusif quasipermanent, $C_{\mathrm{L}}{ }^{\mathrm{I}}=C_{\mathrm{L}}{ }^{\infty} / k$, et les équations (31) et (33) permettent de retrouver $\delta=D_{\mathrm{L}} / V_{\mathrm{I}}$. En revanche une convection intense aura pour effet de brasser efficacement le bain de sorte que $\mathrm{C}_{\mathrm{L}}{ }^{\mathrm{I}}$ sera proche de $C_{\mathrm{L}}{ }^{\infty}$, avec pour conséquence une réduction de $\delta$. Il est intéressant de définir un paramètre de convectodiffusion $\Delta=\delta V_{\mathrm{I}} / D$ en normalisant l'épaisseur de la couche limite $\delta$ par sa valeur de référence en

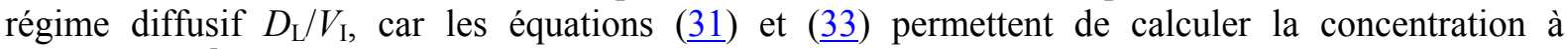
l'interface $C_{\mathrm{L}}{ }^{\mathrm{I}}$ en fonction de $\Delta$ :

$$
C_{\mathrm{L}}^{\mathrm{I}}=C_{\mathrm{L}}^{\infty} /[1-(1-k) \Delta] .
$$

La dernière étape consiste à définir un coefficient de partage effectif $k_{\text {eff }}$ comme le rapport de la composition incorporée dans le solide $C_{\mathrm{S}}{ }^{\mathrm{I}}$ à la composition au loin dans le bain $C_{\mathrm{L}}{ }^{\infty}$. En utilisant l'équation (프), il vient :

$$
k_{\text {eff }}=C_{\mathrm{S}}^{\mathrm{I}} / C_{\mathrm{L}}^{\infty}=k C_{\mathrm{L}}^{\mathrm{I}} / C_{\mathrm{L}}^{\infty}=k /[1-(1-k) \Delta] .
$$

Le coefficient de partage effectif $k_{\text {eff }}$ variant entre $k$ pour les régimes de convection intense $(\Delta \rightarrow 0)$ et 1 pour le régime diffusif $(\Delta=1)$, on retrouve ainsi les cas limites présentés dans les sections 4.3 .1 et 4.3.2. Un des résultats du travail pionnier de Burton, Prim et Schlichter [33] a été de montrer que $k_{\text {eff }}$ pouvait être substitué à $k$ dans l'équation de Scheil-Gulliver (29) pour prédire les profils de ségrégation axiale dans les cristaux élaborés :

$$
C_{\mathrm{S}}(Z)=k_{\mathrm{eff}} C_{0}\left(1-f_{\mathrm{S}}\right)^{(\mathrm{keff}-1)} .
$$


L'équation précédente n'est valable qu'après une phase transitoire nécessaire pour que la concentration $C_{\mathrm{S}}(Z)$ passe de $k C_{0}$ à $k_{\mathrm{eff}} C_{0}$. Par ailleurs, la discussion faite en section 4.3 .1 sur la validité de l'équation de Scheil-Gulliver en fin de solidification pour $k<1$ peut être reprise ici. Signalons pour conclure que le formalisme de couche limite solutale s'est révélé extrêmement riche pour la compréhension des mécanismes de transport. Citons sans pouvoir en dire plus le cas de la ségrégation radiale, qu'on peut associer aux variations de l'épaisseur de la couche le long du front de croissance [28], mais les problèmes de striations de croissance que nous allons maintenant aborder rentrent également dans ce cadre.

\subsubsection{Striations de croissance}

Les striations de croissance sont des variations de composition dont l'origine peut être corrélée à des instabilités des conditions de solidification. Leur périodicité est variable d'une expérience à l'autre, mais reste généralement de l'ordre de la centaine de micromètres. Concernant les sources du phénomène, des problèmes liés au dispositif de solidification, vibrations ou défauts de stabilité de la tête de tirage, peuvent conduire à des fluctuations de la vitesse de croissance qui vont à leur tour induire des différences de concentration dans le matériau. Ce mécanisme est illustré en figure 14 pour un alliage de silicium dopé à l'antimoine [34]. Sur l'exemple proposé, les variations de compositions restent modérées, mais il importe de noter qu'elles peuvent parfois dépasser les $100 \%$ en relatif. Un écoulement convectif instationnaire est également source de striations, soit directement par l'équation (28), soit indirectement, via le couplage avec la thermique du bain.

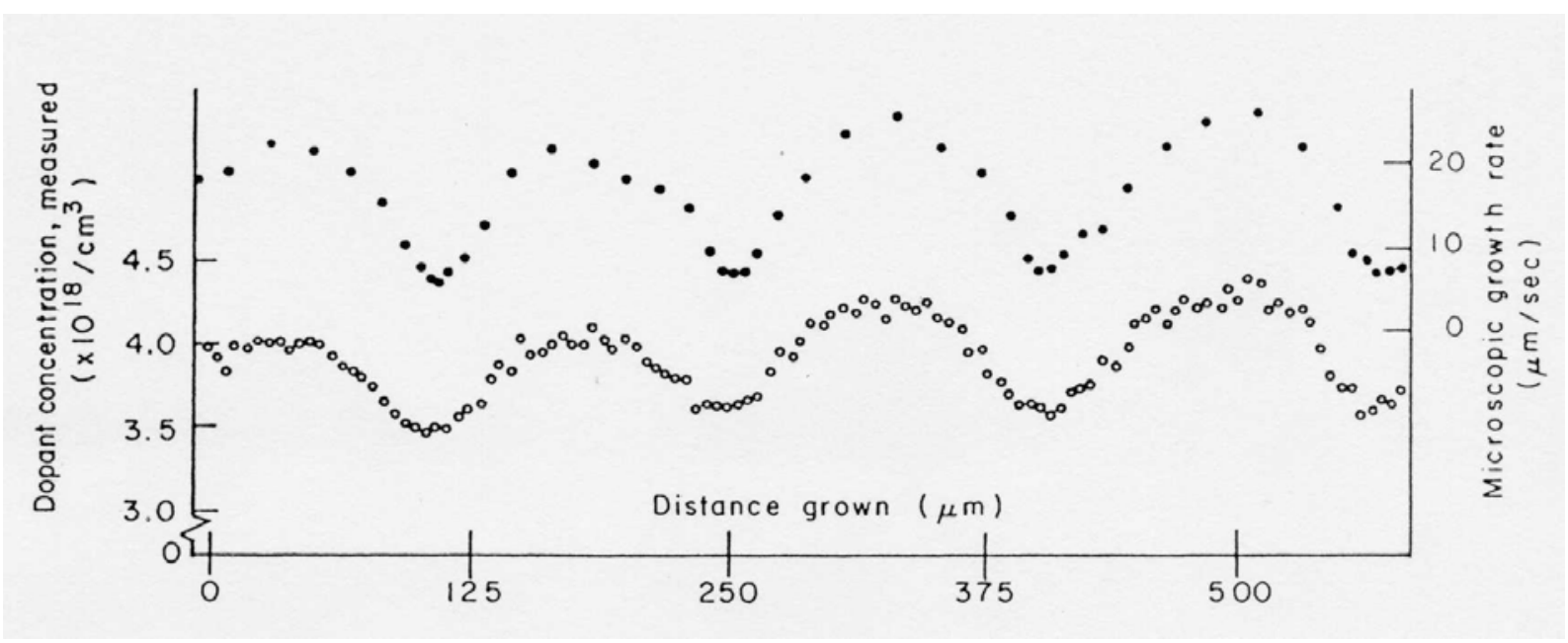

FIG. 14. Corrélation entre les variations de concentration (courbe du bas) et de vitesse locale de solidification (courbe du haut) le long d'un cristal de silicium dopé à l'antimoine [34].

Même en se restreignant à une configuration mono-dimensionnelle, une solution analytique exacte des équations de transport instationnaires est pratiquement impossible à obtenir. Comme sur d'autres problèmes, les simulations numériques ont permis d'obtenir des résultats intéressants [35], mais une meilleure compréhension des mécanismes physiques passe également par la recherche de solutions analytiques approchées. Une façon pour ce faire est de considérer l'équation de référence $(\underline{28}) \mathrm{du}$ transport solutal en y ajoutant des termes de la forme $V_{\mathrm{I}} \exp (i \omega t) \partial C_{\mathrm{L}} / \partial Z$ ou $V_{\mathrm{C}} \exp (i \omega t) \partial C_{\mathrm{L}} / \partial Z$ selon que la perturbation instationnaire est une vitesse d'interface ou une vitesse convective. Il est alors possible d'étudier la réponse du champ de concentration, qu'on peut supposer linéaire dans l'hypothèse où ces termes perturbateurs restent d'amplitude faible.

D'un point de vue physique, il est naturel de penser que le champ de concentration ne pourra pas suivre les variations trop rapides. On peut même estimer la fréquence de coupure du système par une analyse des ordres de grandeur des termes de l'équation de transport. En posant $\partial C_{\mathrm{L}} / \partial t \approx \omega\left(C_{\mathrm{L}}{ }^{\mathrm{I}}-C_{\mathrm{L}}{ }^{\infty}\right)$ et $D_{\mathrm{L}} \partial^{2} C_{\mathrm{L}} / \partial Z^{2} \approx\left(D_{\mathrm{L}} / \delta^{2}\right)\left(C_{\mathrm{L}}{ }^{\mathrm{I}}-C_{\mathrm{L}}{ }^{\infty}\right)$ où $\omega$ et $\delta$ représentent respectivement la pulsation de la fluctuation imposée et l'épaisseur de la couche limite solutale, on peut se convaincre que le champ de concentration parviendra à suivre les fluctuations de pulsation inférieure à $D_{\mathrm{L}} / \delta^{2}$. De façon plus générale, la réponse reliant la variation de concentration dans le cristal élaboré et la 
perturbation en entrée prend la forme d'un filtre passe bas dont le gain dépend du paramètre de convecto-diffusion introduit en section $\underline{4.3 .3}$ [36].

Soulignons pour conclure les implications pratiques de cette approche théorique. En prenant des valeurs typiques des paramètres pertinents, $D_{\mathrm{L}}=5.10^{-9} \mathrm{~m}^{2} \cdot \mathrm{s}^{-1}$ et $\delta=10^{-4} \mathrm{~m}$, on voit que la pulsation de coupure sera de l'ordre du Hz. Des perturbations de fréquence plus élevées, comme celles associées aux groupes de pompage, seront donc significativement amorties. En revanche, l'expérimentateur devra porter son attention sur l'optimisation du système de tirage, la régulation de la thermique du dispositif ou la stationnarité de l'écoulement convectif, dans la mesure où les fluctuations induites sont généralement, à très basse fréquence et en tant que telles, sources de striations dans les cristaux élaborés.

\subsection{Déstabilisation morphologique}

La déstabilisation morphologique est un mécanisme permettant la structuration de l'interface de solidification, dans le but d'augmenter sa surface d'échange pour une meilleure évacuation de la chaleur ou du soluté en excès. La formation de cristaux dendritiques dans un bain surfondu est une illustration de ce phénomène. Pour les problèmes de solidification dirigée d'alliages, on sait depuis de nombreuses années qu'un front de croissance initialement lisse peut devenir cellulaire ou dendritique quand la vitesse de tirage dépasse un seuil. Vu son importance en pratique - la formation de structures s'accompagnant d'une dégradation irréversible de la qualité cristalline du matériau - ce problème de déstabilisation morphologique a été très largement étudié dans la littérature, et le lecteur pourra se reporter à l'article de synthèse [37] pour plus d'informations sur le sujet.

Au niveau des mécanismes, le développement ou l'amortissement d'une perturbation peut au premier ordre se comprendre en considérant simultanément le champ thermique imposé par le dispositif et la température locale de fusion, liée aux variations de composition dans la couche limite solutale. La figure 15 montre qu'il est en effet possible d'associer à chaque point du profil de $C_{\mathrm{L}}$, via le diagramme des phases du système, une température locale de fusion $T_{\mathrm{L}}$. La situation représentée en figure 15 est celle d'un régime de transport diffusif, mais le raisonnement est applicable à toutes les configurations de couche limite.

D'autre part, la thermique du dispositif, contrôlée dans une certaine mesure par les choix de l'expérimentateur, gouverne la température $T_{\mathrm{q}}$ à l'avant du front de solidification. Supposons maintenant une fluctuation des conditions de croissance, liée par exemple aux systèmes de régulation ou de tirage, conduisant à la formation d'une avancée locale l'interface. Pour le cas représenté en figure 15 , où existe un domaine tel que $T_{\mathrm{L}}\left(Z_{\mathrm{L}}\right)$ soit supérieur à $T_{\mathrm{q}}\left(Z_{\mathrm{L}}\right)$, le solide ainsi formé est thermodynamiquement stable, et la déformation de l'interface apparaît donc irréversible.

En revanche, si le gradient thermique $G_{\mathrm{T}}$ imposé par le dispositif est suffisamment élevé, le solide formé va être refondu, puisqu'il se retrouve dans un environnement où la phase stable est la phase liquide, avec pour conséquence le retour de l'interface à la planéité. En termes quantitatifs, le critère de stabilité peut donc s'écrire en comparant les gradients de température et de concentration à l'interface côté liquide :

$$
G_{\mathrm{T}}>m_{\mathrm{L}} G_{\mathrm{C}}^{\mathrm{I}}
$$

où $G_{\mathrm{C}}{ }^{\mathrm{I}}$ est donné par l'équation (31) de conservation du soluté à l'interface :

$$
G_{\mathrm{C}}{ }^{\mathrm{I}}=-\left(V_{\mathrm{I}} / D_{\mathrm{L}}\right)(1-k) C_{\mathrm{L}}{ }^{\mathrm{I}} .
$$

Pour le régime de transport solutal diffusif illustré en figure $\underline{15}, C_{\mathrm{L}}{ }^{\mathrm{I}}=C_{0} / k$, alors que dans le cas général on peut écrire $C_{\mathrm{L}}{ }^{\mathrm{I}}=k_{\text {eff }}\left(C_{0} / k\right)$, où $k_{\text {eff }}$ est le coefficient de partage effectif introduit en section 4.3.3. Ce critère de surfusion constitutionnelle, introduit par Tiller et al. [38], permet des prédictions dont l'accord avec les résultats expérimentaux est relativement satisfaisant aux faibles vitesses de tirage. Cela dit, il n'apporte pas d'information sur la longueur d'onde de la structure formée, et ne permet pas de juger de l'influence de l'énergie interfaciale $\sigma_{\mathrm{LS}}$. Dans la mesure où la formation de structures est potentiellement coûteuse du point de vue énergétique, il s'agit d'une limitation sérieuse. 


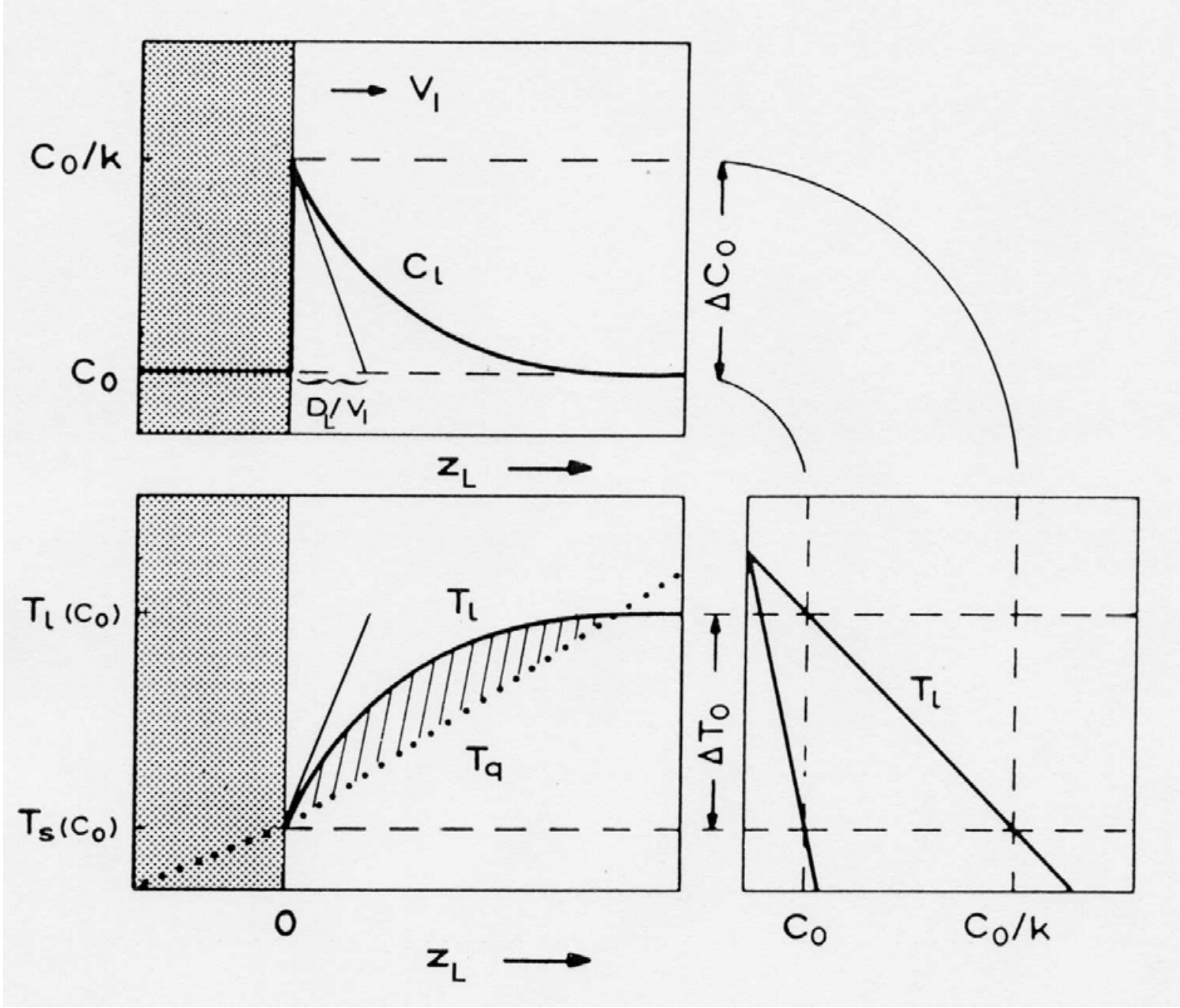

FIG. 15. Variation des températures $T_{\mathrm{q}}$ imposée par le dispositif de croissance et $T_{\mathrm{L}}$ déduite du profil de concentration et du diagramme des phases en fonction de la distance à l'interface côté liquide [4]. La comparaison entre $T_{\mathrm{q}}$ et $T_{\mathrm{L}}$ permet de conclure quant à la stabilité du front de solidification.

Une analyse de stabilité plus complète du point de vue des mécanismes permettant de déterminer le seuil d'instabilité et la longueur d'onde de la structure formée a été proposée par Mullins et Sekerka [32] en 1964. L'idée de base est de déterminer la réponse du système à une perturbation infinitésimale de l'interface de la forme $Z(Y, t)=\varepsilon(t) \sin (\omega Y), Y$ représentant une coordonnée spatiale le long du front de croissance. Outre les équations de bilan sur la thermique et le soluté, l'approche théorique prend en compte l'effet de la courbure de l'interface sur la température d'équilibre solideliquide.

En supposant la réponse du système linéaire, il est possible de calculer la dérivée temporelle $\mathrm{d} \varepsilon / \mathrm{d} t$, un rapport $(\mathrm{d} \varepsilon / \mathrm{d} t) / \varepsilon$ positif correspondant à une situation où la perturbation est amplifiée. La dernière étape est d'utiliser la condition de stabilité marginale $\mathrm{d} \varepsilon / \mathrm{d} t=0$, et le critère de stabilité peut être mis sous la forme [39] :

$$
\left(G_{\mathrm{T}} / V_{\mathrm{I}}\right)+1 / 2\left(\Delta H_{\mathrm{V}} / K_{\mathrm{L}}\right)>-1 / 2\left[\left(K_{\mathrm{S}}+K_{\mathrm{L}}\right) / K_{\mathrm{L}}\right]\left(m_{\mathrm{L}} / D_{\mathrm{L}}\right)(1-k) C_{\mathrm{L}}{ }^{\mathrm{I}} S(A),
$$

où $K_{\mathrm{S}}+K_{\mathrm{L}}$ représentent respectivement les conductivités thermiques des phases solide et liquide, la chaleur latente de solidification $\Delta H_{\mathrm{V}}$ étant exprimée en $\mathrm{J} / \mathrm{m}^{3}$. La fonction de stabilité de Sekerka $S(A)$ permet de rendre compte de l'effet de l'énergie interfaciale, qui apparaît dans le paramètre adimensionnel $A$, défini par [푸] :

$$
A=-\left[\sigma_{\mathrm{LS}} k^{2} V_{\mathrm{I}} T_{\mathrm{F}}\right] /\left[m_{\mathrm{L}} D_{\mathrm{L}} \Delta H_{\mathrm{V}}(1-k) C_{0}\right] .
$$




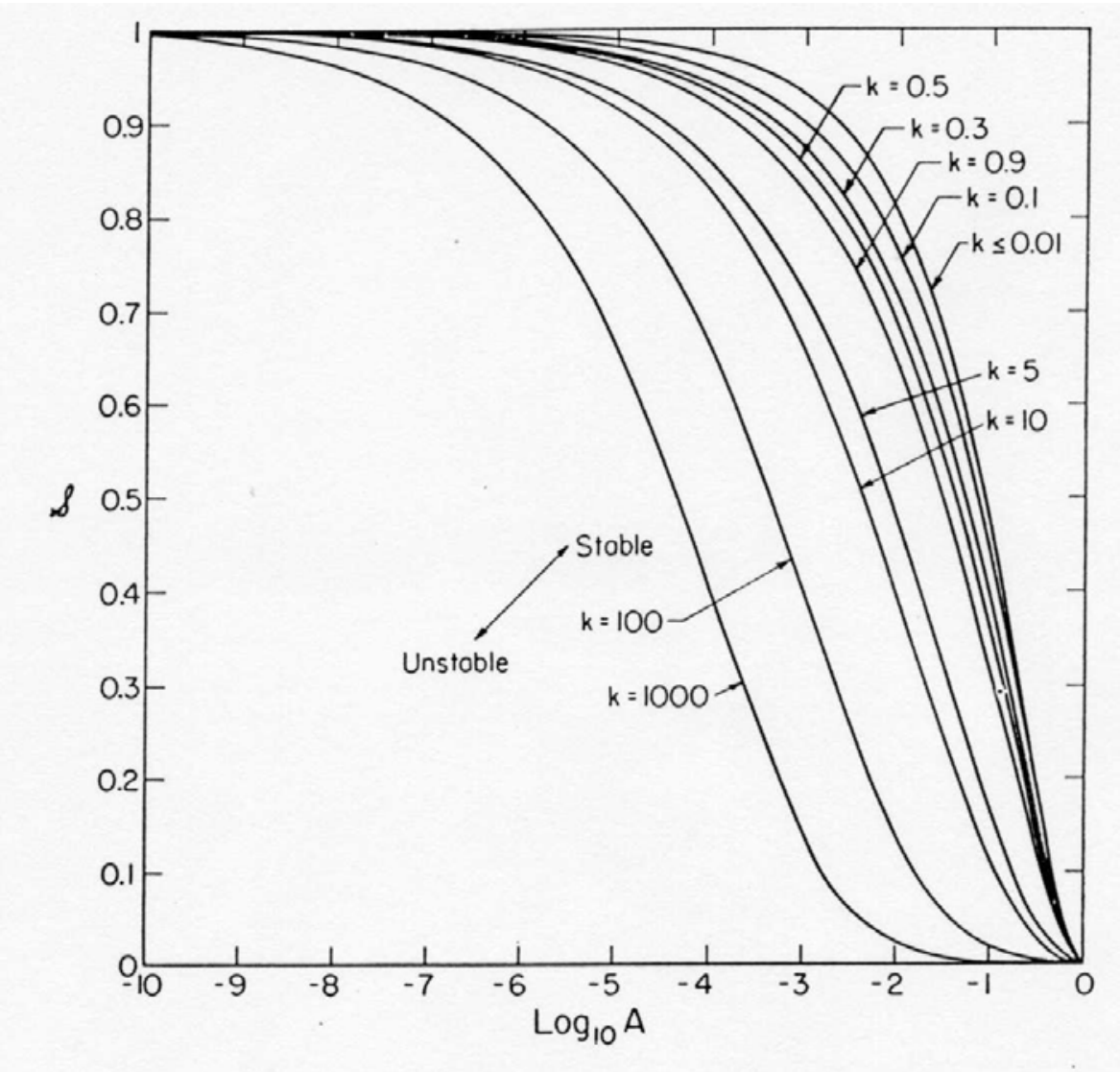

FIG. 16. Variation de la fonction de stabilité de Sekerka en fonction du paramètre adimensionnel $A$ défini par l'équation $(\underline{40})[\underline{39}]$.

La fonction de Sekerka est représentée sous forme graphique sur la figure 16. Il est intéressant de remarquer que quand ce paramètre $A$ tend vers l'unité, $S(A)$ tend vers zéro et l'interface devient inconditionnellement stable. Le mécanisme physique sous-jacent est que le coût énergétique de formation de la structure est prohibitif dans la mesure où la longueur d'onde prédite par la théorie est très petite. Pour des valeurs conventionnelles des paramètres thermophysiques de l'alliage, ce comportement peut être observé aux fortes vitesses de solidification, de l'ordre du m.s ${ }^{-1}$.

En revanche, aux vitesses classiquement utilisées en cristallogenèse, le paramètre $A$ prend des valeurs très faibles, et $S(A)$ reste proche de l'unité. Dans ce cas, l'effet de l'énergie interfaciale sur le seuil de stabilité est tout à fait négligeable. Une analyse des ordres de grandeur permet également de montrer que la contribution du terme de chaleur latente est généralement limitée. Sous ces hypothèses, l'équation ( $\underline{39}$ ) peut se mettre sous une forme très similaire au critère de surfusion constitutionnelle :

$$
2\left[K_{\mathrm{L}} /\left(K_{\mathrm{S}}+K_{\mathrm{L}}\right)\right] G_{\mathrm{T}}>-m_{\mathrm{L}}\left(V_{\mathrm{I}} / D_{\mathrm{L}}\right)(1-k) C_{\mathrm{L}}^{\mathrm{I}},
$$

la seule différence étant que le gradient thermique est maintenant pondéré en fonction du rapport des conductivités thermiques en phase solide et liquide. Pour tous les matériaux où $K_{\mathrm{S}}$ et $K_{\mathrm{L}}$ sont proches, le résultat précédent, associé à une inévitable imprécision sur les données de diffusivité, permet d'expliquer le succès du critère de surfusion constitutionnelle pour les vitesses de tirage modérées.

Dans la pratique, il est toutefois préférable d'utiliser la forme modifiée donnée par l'équation (39), en tenant compte de la chaleur latente et de l'énergie interfaciale. D'autres mécanismes physiques ont été invoqués pour prédire des modifications du seuil de stabilité [37], mais leur étude dépasse le cadre de ce texte. Rappelons enfin que, comme toute analyse de stabilité linéaire, l'approche de Mullins et Sekerka n'est valide qu'au voisinage du seuil, le comportement exponentiel du développement de la perturbation nécessitant ensuite la prise en compte des termes non-linéaires. Ce problème de formation des structures a été largement étudié dans la littérature, en particulier dans le domaine de la métallurgie [4], mais il se situe hors du contexte de la cristallogenèse. 


\section{CONCLUSION : RETOUR VERS L'EXPÉRIENCE}

Il peut être intéressant à ce stade de revenir sur un certain nombre des thèmes rencontrés au cours de cette brève présentation, non plus en termes de mécanismes physiques comme nous l'avons fait jusqu'ici, mais plutôt selon leurs implications expérimentales. Pour les configurations de cristallogenèse, les problèmes les plus fondamentaux sont ceux liés à la thermique de l'installation : l'optimisation du champ de température dans l'échantillon en cours de croissance, entre autres via l'utilisation des techniques de simulation numérique, est une nécessité pour fournir aux utilisateurs des cristaux de qualité satisfaisante. Cette optimisation permet en particulier d'éviter la présence de points froids sources de germinations parasites aux endroits stratégiques du dispositif, et de contrôler les gradients de température pour limiter les contraintes et les densités de dislocations dans la phase solide.

Les problèmes liés à la présence de solutés dans les systèmes polyconstitués sont également très importants en pratique : le paramètre expérimental clé est la vitesse de croissance du cristal, qui doit d'abord être choisie pour éviter la déstabilisation morphologique du front de croissance. Cette vitesse contrôle également dans une certaine mesure le mode de transport du soluté et par suite la ségrégation chimique dans l'échantillon et le niveau des contraintes mécaniques et de densités et de défauts cristallins liés à l'existence de gradients de concentration. Il importe donc de définir, en liaison avec les utilisateurs des cristaux élaborés, le niveau de ségrégation acceptable pour une application donnée.

Les problèmes liés à la présence d'un conteneur nécessaire pour le confinement de la phase liquide peuvent également être très contraignants. Le matériau du creuset peut en effet être source de germination hétérogène et de pollution chimique. Il peut également, en cas de réaction avec le cristal en cours de croissance, être à l'origine de contraintes mécaniques et de défauts cristallins au refroidissement. Ces problèmes sont particulièrement sensibles en configuration Bridgman, et les possibilités d'encapsulation par sel fondu ou film de gaz sont à étudier au cas par cas. Signalons enfin que l'utilisation d'un germe permettant d'orienter la croissance et de s'affranchir de la phase initiale de surfusion dans le bain est toujours à recommander dans la pratique.

L'interaction entre ces différents problèmes nécessite d'identifier des compromis pas toujours évidents entre productivité et qualité cristalline. Par exemple, imposer un gradient de température élevé permet de choisir une vitesse de tirage rapide, mais conduit d'un autre côté à un niveau de contraintes mécaniques et de défauts cristallins important. Avec ce type de gradient, le risque d'instationnarités convectives et de striations dans les cristaux n'est par ailleurs pas à exclure. Un autre exemple de compromis est le choix du creuset, les matériaux type platine ou iridium optimaux du point de vue de l'interaction avec le cristal en cours de croissance étant souvent très onéreux.

Quoi qu'il en soit, le développement des formalismes de la thermodynamique et de la mécanique des milieux continus appliqués aux problématiques de cristallogenèse fait que l'expérimentateur dispose maintenant d'un cadre conceptuel relativement solide. À ce titre, les progrès en termes de compréhension des mécanismes réalisés depuis les années 1950, début de la réflexion sur le sujet, sont certainement appréciables. Cette évolution devrait se poursuivre avec le développement des capacités de calcul des ordinateurs et des méthodes de modélisation numérique, mais il serait pour autant téméraire de prétendre que tous les problèmes pourront être facilement résolus.

Le cas des striations de croissance, développé en section 4.3.4 est à cet égard très significatif : il s'agit d'un domaine où le théoricien peut proposer des pistes d'amélioration, souligner l'intérêt de techniques permettant d'amortir les instabilités convectives ou de créer des barrières thermiques pour filtrer les fluctuations de température. Cela posé, on voit mal comment éviter les striations de croissance en tirage Czochralski... On pourrait multiplier les exemples, mais la conclusion serait souvent la même : les progrès théoriques peuvent orienter les choix du praticien, mais le rêve d'une production automatisée relève encore de la science fiction. En ce sens, la croissance cristalline devrait pour longtemps encore rester un art. 


\section{REMERCIEMENTS}

L'auteur remercie chaleureusement ses collègues B. Drevet, T. Duffar, M.D. Dupouy, D. Camel et B. Vinet pour leur relecture attentive du manuscrit et pour leurs nombreux commentaires sur les sujets abordés dans ce texte. Il tient également à reconnaître le soutien longtemps renouvelé du CNES à l'activité cristallogenèse du laboratoire d'étude de la solidification et la volonté stratégique du CEA qui a permis de soutenir cette activité.

\section{RÉFÉRENCES}

[1] J.C. Brice, The growth of crystals from the melt, North Holland (1965).

[2] F. Dupret et N. Van den Bogaert, Modelling Bridgman and Czochralski growth, in Handbook of Crystal Growth, Vol. 2, D.T.J. Hurle ed., North Holland (1994).

[3] B. Mutaftschiev, Nucleation theory, in Handbook of Crystal Growth, Vol. 1, D.T.J. Hurle ed., North Holland (1993).

[4] W. Kurz et D.J. Fisher, Fundamentals of solidification, Trans Tech Publications (1989).

[5] D. Turnbull et J.C. Fisher, J. Chem. Phys. 17 (1949) 71.

[6] P.J. Desré, A.R. Yavari et P. Hicter, Phil. Mag. 61 (1990) 3.

[7] M. Grant et J.D. Gunton, Phys. Rev. B 32 (1985) 7299.

[8] D.H. Rasmussen, J. Crystal Growth 56 (1982) 45.

[9] B. Vinet, L. Magnusson, H. Fredriksson et P.J. Desré, soumis à Int. J. Non-Equil. Process.

[10] P. Bennema, Growth and morphology of crystals, in Handbook of Crystal Growth, Vol. 1, D.T.J. Hurle ed., North Holland (1993).

[11] M.C. Flemmings, Solidification processing, Mc Graw-Hill Inc. (1974).

[12] K.A. Jackson, in Liquid Metals and Solidification, American Society of Metals (1958).

[13] J.W. Cahn, Acta Met. 8 (1960) 554.

[14] W.K. Burton, N. Cabrera et F.C. Frank, Phil. Trans. Roy. Soc. A243 (1951) 299.

[15] R.J. Roedel, A.R. Von Neida et R. Caruso, J. Electrochem. Soc. 126 (1979) 637.

[16] A.W. Rempel et M.G. Worster, J. Crystal Growth 205 (1999) 427.

[17] J. Völkl, Stress in the cooling crystal, in Handbook of Crystal Growth, Vol. 2, D.T.J. Hurle ed., North Holland (1994).

[18] T. Duffar, J.M. Gourbil, P. Boiton, P. Dusserre et N. Eustathopoulos, J. Crystal Growth 179 (1997) 356.

[19] J.P. Garandet, J. Crystal Growth 96 (1989) 680.

[20] A.S. Jordan, A.R. Von Neida et R. Caruso, J. Crystal Growth 76 (1986) 243.

[21] H. Alexander et P. Haasen, Solid State Phys. 22 (1968) 28.

[22] J.P. Hirth et J. Lothe, Theory of dislocations, $2^{\text {ème }}$ edition, Wiley (1982).

[23] N. Miyazaki et S. Okuyama, J. Crystal Growth 183 (1998) 81.

[24] Binary Alloy Phase Diagrams, $2^{\text {ème }}$ edition. T.B. Massalski ed., The Materials Information Society (1990).

[25] D. DeNobel, Philips Research Repts 14 (1959) 361.

[26] I. Ansara, M. Gambino et J.P. Bros, J. Crystal Growth 32 (1976) 101.

[27] E. Brück, R.J. Raymakers, R.K. Route et R.S. Feigelson, J. Crystal Growth 128 (1993) 933. 
[28] J.P. Garandet, J.J. Favier et D. Camel, Segregation phenomena in Crystal growth from the melt, in Handbook of Crystal Growth, Vol. 2, D.T.J. Hurle ed., North Holland (1994).

[29] D. Schwabe, Surface tension driven flow in crystal growth melts, in Crystals: Growth, Properties and Applications, Springer Verlag, 11 (1988) 75.

[30] P. Dold et K.W. Benz, Rotating magnetic field: fluid flow and crystal growth applications, Progress in Crystal Growth and Characterization of Materials 38 (1999) 7.

[31] W.G. Pfann, Zone Melting, $2^{\text {ème }}$ edition. Wiley (1966).

[32] W.W. Mullins et R.F. Sekerka, J. Appl. Phys. 35 (1964) 444.

[33] J.A. Burton, R.C. Prim et W.P. Schlichter, J. Chem. Phys. 21 (1953) 1987.

[34] A. Murgai, H.C. Gatos et A.F. Witt, J. Electrochem. Soc. 123 (1976) 224.

[35] T. Jung et G. Müller, J. Crystal Growth 171 (1997) 373.

[36] F.Z. Haddad, J.P. Garandet, D. Henry et H. Ben Hadid, J. Crystal Growth 204 (1999) 213.

[37] S.R. Coriell et G.B. Mc Fadden, Morphological Stability, in Handbook of Crystal Growth, Vol. 1, D.T.J. Hurle ed., North Holland (1993).

[38] W.A. Tiller, K.A. Jackson, J.W. Rutter et B. Chalmers, Acta Met. 1 (1953) 428.

[39] R.F. Sekerka, J. Crystal Growth, 3/4 (1968) 71. 